\title{
Generalized Guglielmo Numbers: An Investigation of Properties of Triangular, Oblong and Pentagonal Numbers via Their Third Order Linear Recurrence Relations
}

\author{
Yüksel Soykan
}

\begin{abstract}
Department of Mathematics, Art and Science Faculty, Zonguldak Bülent Ecevit University, 67100, Zonguldak, Turkey e-mail: yuksel_soykan@hotmail.com
\end{abstract}

\begin{abstract}
In this paper, we investigate the generalized Guglielmo sequences and we deal with, in detail, four special cases, namely, triangular, triangular-Lucas, oblong and pentagonal sequences. We present Binet's formulas, generating functions, Simson formulas, and the summation formulas for these sequences. Moreover, we give some identities and matrices related with these sequences.
\end{abstract}

\section{Introduction}

An oblong (or promic, or pronic, or heteromecic) number $O_{n}$ is a number which is the product of two consecutive integers, that is, a number of the form $O_{n}=$ $n(n+1)$. Moreover, the nth oblong number is the sum of the first $n$ even integers, i.e.,

$$
O_{n}=\sum_{k=1}^{n} 2 k=n(n+1) .
$$

The study of these numbers dates back to Aristotle. The first few oblong numbers are:

$$
0,2,6,12,20,30,42,56,72,90,110,132,156,182,210,240,272,306,342,380,420,462, \ldots
$$

Received: December 27, 2021; Accepted: February 9, 2022

2020 Mathematics Subject Classification: 11B37, 11B39, $11 B 83$.

Keywords and phrases: generalized Guglielmo numbers, Tribonacci numbers, triangular numbers, triangular-Lucas numbers, oblong numbers, pentagonal numbers. 
(sequence A002378 in the OEIS [18). The triangular number is half of the oblong number, i.e.,

$$
T_{n}=\sum_{k=1}^{n} k=\frac{n(n+1)}{2}=\frac{O_{n}}{2} .
$$

The first few triangular numbers are:

$0,1,3,6,10,15,21,28,36,45,55,66,78,91,105,120,136,153,171,190,210,231, \ldots$

(sequence A000217 in the OEIS). Oblong and triangular sequences have been studied by many authors and more detail can be found in the extensive literature

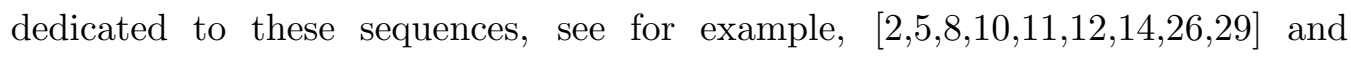
references therein. For more references, see the sequences A002378 and A000217 in the OEIS. Note that oblong and triangular sequences have the following properties:

$$
\begin{aligned}
T_{n}+T_{n+1} & =(n+1)^{2}, \\
\sum_{k=1}^{n} T_{k} & =\frac{n(n+1)(n+2)}{6}, \\
T_{m+n} & =T_{m}+T_{n}+m n, \\
T_{m n} & =T_{m} T_{n}+T_{m-1} T_{n-1}, \\
T_{n}^{2} & =T_{n}+T_{n-1} T_{n+1}, \\
T_{n} & =T_{n-1}+n, \\
O_{n} & =O_{n-1}+2 n, \\
O_{T_{n}} & =O_{n}+O_{T_{n}-1} .
\end{aligned}
$$

A pentagonal number is given by the formula

$$
p_{n}=\frac{1}{2} n(3 n-1) .
$$

The first few pentagonal numbers are:

$$
0,1,5,12,22,35,51,70,92,117,145,176,210,247,287,330,376,425,477,532,590,651, \ldots
$$


(sequence A000326 in the OEIS). Note that pentagonal sequence hold the following properties:

$$
\begin{aligned}
& p_{n}=p_{n-1}+3 n-2=2 p_{n-1}-p_{n-2}+3, \\
& p_{n}=T_{n-1}+n^{2}=T_{n}+2 T_{n-1}=T_{2 n-1}-T_{n-1} .
\end{aligned}
$$

A brief introduction on pentagonal numbers can be found in MathWorld 27] and Wikipedia 28.

The sequences $\left\{O_{n}\right\},\left\{T_{n}\right\}$ and $\left\{p_{n}\right\}$ satisfy the following third order linear recurrences:

$$
\begin{aligned}
& O_{n}=3 O_{n-1}-3 O_{n-2}+O_{n-3}, \quad O_{0}=0, O_{1}=2, O_{2}=6, \\
& T_{n}=3 T_{n-1}-3 T_{n-2}+T_{n-3}, \quad T_{0}=0, T_{1}=1, T_{2}=3, \\
& p_{n}=3 p_{n-1}-3 p_{n-2}+p_{n-3}, \quad p_{0}=0, p_{1}=1, p_{2}=5 \text {. }
\end{aligned}
$$

The purpose of this article is to generalize and investigate these interesting sequence of numbers (i.e., oblong, triangular and pentagonal numbers). First, we recall some properties of generalized Tribonacci numbers.

The generalized $(r, s, t)$ sequence (or generalized Tribonacci sequence or generalized 3 -step triangular sequence)

$$
\left\{W_{n}\left(W_{0}, W_{1}, W_{2} ; r, s, t\right)\right\}_{n \geq 0}
$$

(or shortly $\left\{W_{n}\right\}_{n \geq 0}$ ) is defined as follows:

$$
W_{n}=r W_{n-1}+s W_{n-2}+t W_{n-3}, \quad W_{0}=a, W_{1}=b, W_{2}=c, \quad n \geq 3
$$

where $W_{0}, W_{1}, W_{2}$ are arbitrary complex (or real) numbers and $r, s, t$ are real numbers.

This sequence has been studied by many authors, see for example

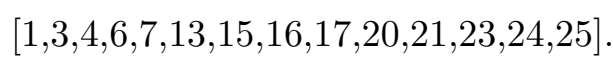

The sequence $\left\{W_{n}\right\}_{n \geq 0}$ can be extended to negative subscripts by defining

$$
W_{-n}=-\frac{s}{t} W_{-(n-1)}-\frac{r}{t} W_{-(n-2)}+\frac{1}{t} W_{-(n-3)}
$$


for $n=1,2,3, \ldots$ when $t \neq 0$. Therefore, recurrence 1 1.1 holds for all integer $n$.

As $\left\{W_{n}\right\}$ is a third-order recurrence sequence (difference equation), its characteristic equation is

$$
x^{3}-r x^{2}-s x-t=0
$$

whose roots are

$$
\begin{aligned}
& \alpha=\alpha(r, s, t)=\frac{r}{3}+A+B, \\
& \beta=\beta(r, s, t)=\frac{r}{3}+\omega A+\omega^{2} B, \\
& \gamma=\gamma(r, s, t)=\frac{r}{3}+\omega^{2} A+\omega B,
\end{aligned}
$$

where

$$
\begin{aligned}
& A=\left(\frac{r^{3}}{27}+\frac{r s}{6}+\frac{t}{2}+\sqrt{\Delta}\right)^{1 / 3}, B=\left(\frac{r^{3}}{27}+\frac{r s}{6}+\frac{t}{2}-\sqrt{\Delta}\right)^{1 / 3}, \\
& \Delta=\Delta(r, s, t)=\frac{r^{3} t}{27}-\frac{r^{2} s^{2}}{108}+\frac{r s t}{6}-\frac{s^{3}}{27}+\frac{t^{2}}{4}, \omega=\frac{-1+i \sqrt{3}}{2}=\exp (2 \pi i / 3) .
\end{aligned}
$$

In the case of single root, i.e., $\alpha=\beta=\gamma$, Binet's formula can be given as follows:

Theorem 1. (Single Root Case: $\alpha=\beta=\gamma$ ) Binet's formula of generalized Fibonacci numbers is

$$
W_{n}=\left(A_{1}+A_{2} n+A_{3} n^{2}\right) \times \alpha^{n}
$$

where

$$
\begin{aligned}
& A_{1}=W_{0}, \\
& A_{2}=\frac{1}{2}\left(-W_{2}+4 W_{1}-3 W_{0}\right), \\
& A_{3}=\frac{1}{2}\left(W_{2}-2 W_{1}+W_{0}\right) .
\end{aligned}
$$

Next, we give the ordinary generating function $\sum_{n=0}^{\infty} W_{n} x^{n}$ of the sequence $W_{n}$. 
Lemma 2. Suppose that $f_{W_{n}}(x)=\sum_{n=0}^{\infty} W_{n} x^{n}$ is the ordinary generating function of the generalized $(r, s, t)$ sequence (the generalized Tribonacci sequence) $\left\{W_{n}\right\}_{n \geq 0}$. Then, $\sum_{n=0}^{\infty} W_{n} x^{n}$ is given by

$$
\sum_{n=0}^{\infty} W_{n} x^{n}=\frac{W_{0}+\left(W_{1}-r W_{0}\right) x+\left(W_{2}-r W_{1}-s W_{0}\right) x^{2}}{1-r x-s x^{2}-t x^{3}} .
$$

Matrix formulation of $W_{n}$ can be given as

$$
\left(\begin{array}{c}
W_{n+2} \\
W_{n+1} \\
W_{n}
\end{array}\right)=\left(\begin{array}{lll}
r & s & t \\
1 & 0 & 0 \\
0 & 1 & 0
\end{array}\right)^{n}\left(\begin{array}{l}
W_{2} \\
W_{1} \\
W_{0}
\end{array}\right)
$$

For matrix formulation (1.5), see [9. In fact, Kalman gave the formula in the following form

$$
\left(\begin{array}{c}
W_{n} \\
W_{n+1} \\
W_{n+2}
\end{array}\right)=\left(\begin{array}{lll}
0 & 1 & 0 \\
0 & 0 & 1 \\
r & s & t
\end{array}\right)^{n}\left(\begin{array}{l}
W_{0} \\
W_{1} \\
W_{2}
\end{array}\right)
$$

Now, we present Simson's formula of generalized Tribonacci numbers.

Theorem 3 (Simson's Formula of Generalized Tribonacci Numbers). For all integers $n$, we have

$$
\left|\begin{array}{ccc}
W_{n+2} & W_{n+1} & W_{n} \\
W_{n+1} & W_{n} & W_{n-1} \\
W_{n} & W_{n-1} & W_{n-2}
\end{array}\right|=t^{n}\left|\begin{array}{ccc}
W_{2} & W_{1} & W_{0} \\
W_{1} & W_{0} & W_{-1} \\
W_{0} & W_{-1} & W_{-2}
\end{array}\right| .
$$

Proof. For a proof, see Soykan 19.

Next, we consider two special cases of the generalized $(r, s, t)$ sequence $\left\{W_{n}\right\}$ which we call them $(r, s, t)$ and Lucas $(r, s, t)$ sequences. $(r, s, t)$ sequence $\left\{G_{n}\right\}_{n \geq 0}$ and Lucas $(r, s, t)$ sequence $\left\{H_{n}\right\}_{n \geq 0}$ are defined, respectively, by the third-order recurrence relations

$$
\begin{array}{ll}
G_{n+3}=r G_{n+2}+s G_{n+1}+t G_{n}, & G_{0}=0, G_{1}=1, G_{2}=r \\
H_{n+3}=r H_{n+2}+s H_{n+1}+t H_{n}, & H_{0}=3, H_{1}=r, H_{2}=2 s+r^{2}
\end{array}
$$


The sequences $\left\{G_{n}\right\}_{n \geq 0}$ and $\left\{H_{n}\right\}_{n \geq 0}$ can be extended to negative subscripts by defining

$$
\begin{aligned}
G_{-n} & =-\frac{s}{t} G_{-(n-1)}-\frac{r}{t} G_{-(n-2)}+\frac{1}{t} G_{-(n-3)}, \\
H_{-n} & =-\frac{s}{t} H_{-(n-1)}-\frac{r}{t} H_{-(n-2)}+\frac{1}{t} H_{-(n-3)}
\end{aligned}
$$

for $n=1,2,3, \ldots$ respectively. Therefore, recurrences (1.7)-1.8 hold for all integers $n$.

For all integers $n,(r, s, t)$, Lucas $(r, s, t)$ numbers can be expressed using Binet's formulas as

$$
\begin{aligned}
G_{n} & =\frac{\alpha^{n+1}}{(\alpha-\beta)(\alpha-\gamma)}+\frac{\beta^{n+1}}{(\beta-\alpha)(\beta-\gamma)}+\frac{\gamma^{n+1}}{(\gamma-\alpha)(\gamma-\beta)}, \\
H_{n} & =\alpha^{n}+\beta^{n}+\gamma^{n}
\end{aligned}
$$

respectively.

In the case of two distinct roots, i.e., $\alpha=\beta \neq \gamma$, for all integers $n$, Binet's formula of $(r, s, t)$ and Lucas $(r, s, t)$ numbers (using initial conditions in (1.7)-(1.8) can be expressed as follows:

Theorem 4. (Single Root Case: $\alpha=\beta=\gamma$ ) For all integers n, Binet's formula of $(r, s, t)$ and Lucas $(r, s, t)$ numbers are

$$
\begin{aligned}
G_{n} & =\frac{1}{2}\left((-3 \alpha+4) n+(-3 \alpha-2) n^{2}\right) \times \alpha^{n}, \\
H_{n} & =\frac{3}{2}\left(2-(\alpha-1)(\alpha-3) n+(\alpha-1)^{2} n^{2}\right) \times \alpha^{n},
\end{aligned}
$$

respectively.

Lemma 2 gives the following results as particular examples (generating functions of $(r, s, t)$ and Lucas $(r, s, t)$ numbers).

Corollary 5. Generating functions of $(r, s, t)$ and Lucas $(r, s, t)$ numbers are

$$
\begin{aligned}
\sum_{n=0}^{\infty} G_{n} x^{n} & =\frac{x}{1-r x-s x^{2}-t x^{3}}, \\
\sum_{n=0}^{\infty} H_{n} x^{n} & =\frac{3-2 r x-s x^{2}}{1-r x-s x^{2}-t x^{3}},
\end{aligned}
$$


respectively.

The following theorem shows that the generalized Tribonacci sequence $W_{n}$ at negative indices can be expressed by the sequence itself at positive indices.

Theorem 6. For $n \in \mathbb{Z}$, we have

$$
W_{-n}=t^{-n}\left(W_{2 n}-H_{n} W_{n}+\frac{1}{2}\left(H_{n}^{2}-H_{2 n}\right) W_{0}\right) .
$$

Proof. For the proof, see Soykan 22, Theorem 2].

Now, we present a basic relation between $\left\{H_{n}\right\}$ and $\left\{W_{n}\right\}$ which can be used to write $H_{n}$ in terms of $W_{n}$.

Lemma 7. The following equality is true:

$\left(W_{2}^{3}+(t+r s) W_{1}^{3}+t^{2} W_{0}^{3}+\left(r^{2}-s\right) W_{1}^{2} W_{2}-2 r W_{1} W_{2}^{2}-s W_{0} W_{2}^{2}+r t W_{0}^{2} W_{2}+\left(s^{2}+\right.\right.$ $\left.r t) W_{0} W_{1}^{2}+2 s t W_{0}^{2} W_{1}+(r s-3 t) W_{0} W_{1} W_{2}\right) H_{n}=\left(3 W_{2}^{2}+\left(r^{2}-s\right) W_{1}^{2}+r t W_{0}^{2}-\right.$ $\left.4 r W_{1} W_{2}-2 s W_{0} W_{2}+(r s-3 t) W_{0} W_{1}\right) W_{n+2}+\left(-2 r W_{2}^{2}+3 t W_{1}^{2}-2 s W_{1} W_{2}-3 t W_{0} W_{2}+\right.$ $\left.3 r s W_{1}^{2}+2 s t W_{0}^{2}+2 r^{2} W_{1} W_{2}+2 s^{2} W_{0} W_{1}+r s W_{0} W_{2}+2 r t W_{0} W_{1}\right) W_{n+1}+\left(-s W_{2}^{2}+\right.$ $\left.\left(s^{2}+r t\right) W_{1}^{2}+3 t^{2} W_{0}^{2}+(r s-3 t) W_{1} W_{2}+2 r t W_{0} W_{2}+4 s t W_{0} W_{1}\right) W_{n}$.

Proof. It is given in Soykan 21.

Using Theorem 6, we have the following corollary, see Soykan 22, Corollary $6]$.

Corollary 8. For $n \in \mathbb{Z}$, we have

(a) $G_{-n}=\frac{1}{t^{n+1}}\left(\left(2 r t-s^{2}\right) G_{n}^{2}+t G_{2 n}+s G_{n+2} G_{n}-(3 t+r s) G_{n+1} G_{n}\right)$.

(b) $H_{-n}=\frac{1}{2 t^{n}}\left(H_{n}^{2}-H_{2 n}\right)$.

Note that $G_{-n}$ and $H_{-n}$ can be given as follows by using $G_{0}=0$ and $H_{0}=3$ in Theorem 6 .

$$
\begin{aligned}
& G_{-n}=t^{-n}\left(G_{2 n}-H_{n} G_{n}+\frac{1}{2}\left(H_{n}^{2}-H_{2 n}\right) G_{0}\right)=t^{-n}\left(G_{2 n}-H_{n} G_{n}\right), \\
& H_{-n}=t^{-n}\left(H_{2 n}-H_{n} H_{n}+\frac{1}{2}\left(H_{n}^{2}-H_{2 n}\right) H_{0}\right)=\frac{1}{2 t^{n}}\left(H_{n}^{2}-H_{2 n}\right),
\end{aligned}
$$

respectively. 


\section{Generalized Guglielmo Sequence}

In this paper, we consider the case $r=3, s=-3, t=1$. A generalized Guglielmo sequence $\left\{W_{n}\right\}_{n \geq 0}=\left\{W_{n}\left(W_{0}, W_{1}, W_{2}\right)\right\}_{n \geq 0}$ is defined by the third-order recurrence relations

$$
W_{n}=3 W_{n-1}-3 W_{n-2}+W_{n-3}
$$

with the initial values $W_{0}=c_{0}, W_{1}=c_{1}, W_{2}=c_{2}$ not all being zero.

The sequence $\left\{W_{n}\right\}_{n \geq 0}$ can be extended to negative subscripts by defining

$$
W_{-n}=3 W_{-(n-1)}-3 W_{-(n-2)}+W_{-(n-3)}
$$

for $n=1,2,3, \ldots$. Therefore, recurrence (2.1) holds for all integer $n$.

Theorem 1 can be used to obtain Binet formula of generalized Guglielmo numbers. Binet formula of generalized Guglielmo numbers can be given as

$$
W_{n}=A_{1}+A_{2} n+A_{3} n^{2}
$$

where

$$
\begin{aligned}
& A_{1}=W_{0}, \\
& A_{2}=\frac{1}{2}\left(-W_{2}+4 W_{1}-3 W_{0}\right), \\
& A_{3}=\frac{1}{2}\left(W_{2}-2 W_{1}+W_{0}\right),
\end{aligned}
$$

i.e.,

$$
W_{n}=W_{0}+\frac{1}{2}\left(-W_{2}+4 W_{1}-3 W_{0}\right) n+\frac{1}{2}\left(W_{2}-2 W_{1}+W_{0}\right) n^{2} .
$$

Here, in Theorem 1, we use the roots $\alpha, \beta, \gamma$ of the cubic equation

$$
x^{3}-3 x^{2}+3 x-1=(x-1)^{3}=0
$$

where $\alpha=\beta=\gamma=1$.

The first few generalized Guglielmo numbers with positive subscript and negative subscript are given in the following Table 1 . 
Table 1: A few generalized Guglielmo numbers.

\begin{tabular}{ccc}
\hline$n$ & $W_{n}$ & $W_{-n}$ \\
\hline 0 & $W_{0}$ & $W_{0}$ \\
1 & $W_{1}$ & $3 W_{0}-3 W_{1}+W_{2}$ \\
2 & $W_{2}$ & $6 W_{0}-8 W_{1}+3 W_{2}$ \\
3 & $W_{0}-3 W_{1}+3 W_{2}$ & $10 W_{0}-15 W_{1}+6 W_{2}$ \\
4 & $3 W_{0}-8 W_{1}+6 W_{2}$ & $15 W_{0}-24 W_{1}+10 W_{2}$ \\
5 & $6 W_{0}-15 W_{1}+10 W_{2}$ & $21 W_{0}-35 W_{1}+15 W_{2}$ \\
6 & $10 W_{0}-24 W_{1}+15 W_{2}$ & $28 W_{0}-48 W_{1}+21 W_{2}$ \\
7 & $15 W_{0}-35 W_{1}+21 W_{2}$ & $36 W_{0}-63 W_{1}+28 W_{2}$ \\
8 & $21 W_{0}-48 W_{1}+28 W_{2}$ & $45 W_{0}-80 W_{1}+36 W_{2}$ \\
9 & $28 W_{0}-63 W_{1}+36 W_{2}$ & $55 W_{0}-99 W_{1}+45 W_{2}$ \\
10 & $36 W_{0}-80 W_{1}+45 W_{2}$ & $66 W_{0}-120 W_{1}+55 W_{2}$ \\
11 & $45 W_{0}-99 W_{1}+55 W_{2}$ & $78 W_{0}-143 W_{1}+66 W_{2}$ \\
12 & $55 W_{0}-120 W_{1}+66 W_{2}$ & $91 W_{0}-168 W_{1}+78 W_{2}$ \\
13 & $66 W_{0}-143 W_{1}+78 W_{2}$ & $105 W_{0}-195 W_{1}+91 W_{2}$ \\
\hline
\end{tabular}

Now we define four special cases of the sequence $\left\{W_{n}\right\}$. Triangular sequence $\left\{T_{n}\right\}_{n \geq 0}$, triangular-Lucas sequence $\left\{H_{n}\right\}_{n \geq 0}$, oblong sequence $\left\{O_{n}\right\}_{n \geq 0}$ and pentagonal sequence $\left\{p_{n}\right\}_{n \geq 0}$ are defined, respectively, by the third-order recurrence relations

$$
\begin{aligned}
& T_{n}=3 T_{n-1}-3 T_{n-2}+T_{n-3}, \quad T_{0}=0, T_{1}=1, T_{2}=3, \\
& H_{n}=3 H_{n-1}-3 H_{n-2}+H_{n-3}, \quad H_{0}=3, H_{1}=3, H_{2}=3 \text {, } \\
& O_{n}=3 O_{n-1}-3 O_{n-2}+O_{n-3}, \quad O_{0}=0, O_{1}=2, O_{2}=6, \\
& p_{n}=3 p_{n-1}-3 p_{n-2}+p_{n-3}, \quad p_{0}=0, p_{1}=1, p_{2}=5 \text {. }
\end{aligned}
$$

The sequences $\left\{T_{n}\right\}_{n \geq 0},\left\{H_{n}\right\}_{n \geq 0},\left\{O_{n}\right\}_{n \geq 0}$ and $\left\{p_{n}\right\}_{n \geq 0}$ can be extended to 
negative subscripts by defining

$$
\begin{aligned}
T_{-n} & =3 T_{-(n-1)}-3 T_{-(n-2)}+T_{-(n-3)}, \\
H_{-n} & =3 H_{-(n-1)}-3 H_{-(n-2)}+H_{-(n-3)}, \\
O_{-n} & =3 O_{-(n-1)}-3 O_{-(n-2)}+O_{-(n-3)}, \\
p_{-n} & =3 p_{-(n-1)}-3 p_{-(n-2)}+p_{-(n-3)},
\end{aligned}
$$

for $n=1,2,3, \ldots$ respectively. Therefore, recurrences (2.3)-2.6 hold for all integer $n$.

$H_{n}$ is the constant sequence (the all 3's sequence) A010701 in 18.

Next, we present the first few values of the Triangular and Triangular-Lucas, oblong and pentagonal numbers with positive and negative subscripts:

Table 2: The first few values of the special third-order numbers with positive and negative subscripts.

\begin{tabular}{ccccccccccccccc}
\hline$n$ & 0 & 1 & 2 & 3 & 4 & 5 & 6 & 7 & 8 & 9 & 10 & 11 & 12 & 13 \\
\hline$T_{n}$ & 0 & 1 & 3 & 6 & 10 & 15 & 21 & 28 & 36 & 45 & 55 & 66 & 78 & 91 \\
$T_{-n}$ & & 0 & 1 & 3 & 6 & 10 & 15 & 21 & 28 & 36 & 45 & 55 & 66 & 78 \\
$H_{n}$ & 3 & 3 & 3 & 3 & 3 & 3 & 3 & 3 & 3 & 3 & 3 & 3 & 3 & 3 \\
$H_{-n}$ & & 3 & 3 & 3 & 3 & 3 & 3 & 3 & 3 & 3 & 3 & 3 & 3 & 3 \\
$O_{n}$ & 0 & 2 & 6 & 12 & 20 & 30 & 42 & 56 & 72 & 90 & 110 & 132 & 156 & 182 \\
$O_{-n}$ & & 0 & 2 & 6 & 12 & 20 & 30 & 42 & 56 & 72 & 90 & 110 & 132 & 156 \\
$p_{n}$ & 0 & 1 & 5 & 12 & 22 & 35 & 51 & 70 & 92 & 117 & 145 & 176 & 210 & 247 \\
$p_{-n}$ & 2 & 7 & 15 & 26 & 40 & 57 & 77 & 100 & 126 & 155 & 187 & 222 & 260 \\
\hline
\end{tabular}

For all integers $n$, triangular, triangular-Lucas, oblong and pentagonal numbers (using initial conditions in 2.2) can be expressed using Binet's formulas 
as

$$
\begin{aligned}
T_{n} & =\frac{n(n+1)}{2}, \\
H_{n} & =3, \\
O_{n} & =n(n+1), \\
p_{n} & =\frac{1}{2} n(3 n-1),
\end{aligned}
$$

respectively.

Next, we give the ordinary generating function $\sum_{n=0}^{\infty} W_{n} x^{n}$ of the sequence $W_{n}$.

Lemma 9. Suppose that $f_{W_{n}}(x)=\sum_{n=0}^{\infty} W_{n} x^{n}$ is the ordinary generating function of the generalized Guglielmo sequence $\left\{W_{n}\right\}_{n \geq 0}$. Then, $\sum_{n=0}^{\infty} W_{n} x^{n}$ is given by

$$
\sum_{n=0}^{\infty} W_{n} x^{n}=\frac{W_{0}+\left(W_{1}-3 W_{0}\right) x+\left(W_{2}-3 W_{1}+3 W_{0}\right) x^{2}}{1-3 x+3 x^{2}-x^{3}} .
$$

Proof. Take $r=3, s=-3, t=1$ in Lemma 2 .

The previous lemma gives the following results as particular examples.

Corollary 10. Generated functions of triangular, triangular-Lucas, oblong and pentagonal numbers are

$$
\begin{aligned}
\sum_{n=0}^{\infty} T_{n} x^{n} & =\frac{x}{1-3 x+3 x^{2}-x^{3}}, \\
\sum_{n=0}^{\infty} H_{n} x^{n} & =\frac{3-6 x+3 x^{2}}{1-3 x+3 x^{2}-x^{3}}, \\
\sum_{n=0}^{\infty} O_{n} x^{n} & =\frac{2 x}{1-3 x+3 x^{2}-x^{3}}, \\
\sum_{n=0}^{\infty} p_{n} x^{n} & =\frac{x+2 x^{2}}{1-3 x+3 x^{2}-x^{3}},
\end{aligned}
$$

respectively. 


\section{Simson Formulas}

There is a well-known Simson Identity (formula) for Fibonacci sequence $\left\{F_{n}\right\}$, namely,

$$
F_{n+1} F_{n-1}-F_{n}^{2}=(-1)^{n}
$$

which was derived first by R. Simson in 1753 and it is now called as Cassini Identity (formula) as well. This can be written in the form

$$
\left|\begin{array}{cc}
F_{n+1} & F_{n} \\
F_{n} & F_{n-1}
\end{array}\right|=(-1)^{n} .
$$

The following theorem gives generalization of this result to the generalized Guglielmo sequence $\left\{W_{n}\right\}_{n \geq 0}$.

Theorem 11 (Simson Formula of Generalized Guglielmo Numbers). For all integers $n$, we have

$$
\left|\begin{array}{ccc}
W_{n+2} & W_{n+1} & W_{n} \\
W_{n+1} & W_{n} & W_{n-1} \\
W_{n} & W_{n-1} & W_{n-2}
\end{array}\right|=-\left(W_{2}-2 W_{1}+W_{0}\right)^{3} .
$$

Proof. Take $r=3, s=-3, t=1$ in Theorem 3 .

The previous theorem gives the following results as particular examples.

Corollary 12. For all integers $n$, Simson formula of triangular, triangular-Lucas, 
oblong and pentagonal numbers are given as

$$
\begin{aligned}
\left|\begin{array}{ccc}
T_{n+2} & T_{n+1} & T_{n} \\
T_{n+1} & T_{n} & T_{n-1} \\
T_{n} & T_{n-1} & T_{n-2}
\end{array}\right| & =-1, \\
\left|\begin{array}{ccc}
H_{n+2} & H_{n+1} & H_{n} \\
H_{n+1} & H_{n} & H_{n-1} \\
H_{n} & H_{n-1} & H_{n-2}
\end{array}\right| & =0, \\
\left|\begin{array}{ccc}
O_{n+2} & O_{n+1} & O_{n} \\
O_{n+1} & O_{n} & O_{n-1} \\
O_{n} & O_{n-1} & O_{n-2}
\end{array}\right| & =-8, \\
\left|\begin{array}{ccc}
p_{n+2} & p_{n+1} & p_{n} \\
p_{n+1} & p_{n} & p_{n-1} \\
p_{n} & p_{n-1} & p_{n-2}
\end{array}\right| & =-27,
\end{aligned}
$$

respectively.

\section{Some Identities}

In this section, we obtain some identities of generalized Guglielmo, triangular, triangular-Lucas, oblong and pentagonal numbers. First, we can give a few basic relations between $\left\{W_{n}\right\}$ and $\left\{T_{n}\right\}$.

Lemma 13. The following equalities are true:

(a) $W_{n}=\left(10 W_{0}-15 W_{1}+6 W_{2}\right) T_{n+4}+\left(37 W_{1}-24 W_{0}-15 W_{2}\right) T_{n+3}+\left(15 W_{0}-\right.$ $\left.24 W_{1}+10 W_{2}\right) T_{n+2}$.

(b) $W_{n}=\left(6 W_{0}-8 W_{1}+3 W_{2}\right) T_{n+3}+\left(21 W_{1}-15 W_{0}-8 W_{2}\right) T_{n+2}+\left(10 W_{0}-15 W_{1}+\right.$ $\left.6 W_{2}\right) T_{n+1}$.

(c) $W_{n}=\left(3 W_{0}-3 W_{1}+W_{2}\right) T_{n+2}+\left(9 W_{1}-8 W_{0}-3 W_{2}\right) T_{n+1}+\left(6 W_{0}-8 W_{1}+3 W_{2}\right) T_{n}$.

(d) $W_{n}=W_{0} T_{n+1}+\left(W_{1}-3 W_{0}\right) T_{n}+\left(3 W_{0}-3 W_{1}+W_{2}\right) T_{n-1}$. 
(e) $W_{n}=W_{1} T_{n}+\left(W_{2}-3 W_{1}\right) T_{n-1}+W_{0} T_{n-2}$.

(f) $\left(W_{0}-2 W_{1}+W_{2}\right)^{3} T_{n}=\left(3 W_{1}^{2}+W_{2}^{2}-W_{0} W_{1}-3 W_{1} W_{2}\right) W_{n+4}+\left(-8 W_{1}^{2}-\right.$ $\left.3 W_{2}^{2}+3 W_{0} W_{1}-W_{0} W_{2}+9 W_{1} W_{2}\right) W_{n+3}+\left(W_{0}^{2}+9 W_{1}^{2}+3 W_{2}^{2}-6 W_{0} W_{1}+\right.$ $\left.3 W_{0} W_{2}-10 W_{1} W_{2}\right) W_{n+2}$.

(g) $\left(W_{0}-2 W_{1}+W_{2}\right)^{3} T_{n}=\left(W_{1}^{2}-W_{0} W_{2}\right) W_{n+3}+\left(W_{0}^{2}-3 W_{0} W_{1}+3 W_{0} W_{2}-\right.$ $\left.W_{1} W_{2}\right) W_{n+2}+\left(3 W_{1}^{2}+W_{2}^{2}-W_{0} W_{1}-3 W_{1} W_{2}\right) W_{n+1}$.

(h) $\left(W_{0}-2 W_{1}+W_{2}\right)^{3} T_{n}=\left(W_{0}^{2}+3 W_{1}^{2}-3 W_{0} W_{1}-W_{1} W_{2}\right) W_{n+2}+\left(W_{2}^{2}-W_{0} W_{1}+\right.$ $\left.3 W_{0} W_{2}-3 W_{1} W_{2}\right) W_{n+1}+\left(W_{1}^{2}-W_{0} W_{2}\right) W_{n}$.

(i) $\left(W_{0}-2 W_{1}+W_{2}\right)^{3} T_{n}=\left(3 W_{0}^{2}+9 W_{1}^{2}+W_{2}^{2}-10 W_{0} W_{1}+3 W_{0} W_{2}-\right.$ $\left.6 W_{1} W_{2}\right) W_{n+1}+\left(-3 W_{0}^{2}+9 W_{0} W_{1}-W_{2} W_{0}-8 W_{1}^{2}+3 W_{2} W_{1}\right) W_{n}+\left(W_{0}^{2}+\right.$ $\left.3 W_{1}^{2}-3 W_{0} W_{1}-W_{1} W_{2}\right) W_{n-1}$.

(j) $\left(W_{0}-2 W_{1}+W_{2}\right)^{3} T_{n}=\left(6 W_{0}^{2}+19 W_{1}^{2}+3 W_{2}^{2}-21 W_{0} W_{1}+8 W_{0} W_{2}-\right.$ $\left.15 W_{1} W_{2}\right) W_{n}+\left(-8 W_{0}^{2}+27 W_{0} W_{1}-9 W_{0} W_{2}-24 W_{1}^{2}+17 W_{1} W_{2}-3 W_{2}^{2}\right) W_{n-1}+$ $\left(3 W_{0}^{2}+9 W_{1}^{2}+W_{2}^{2}-10 W_{0} W_{1}+3 W_{0} W_{2}-6 W_{1} W_{2}\right) W_{n-2}$.

Proof. Note that all the identities hold for all integers $n$. We prove (a). To show (a), writing

$$
W_{n}=a \times T_{n+4}+b \times T_{n+3}+c \times T_{n+2}
$$

and solving the system of equations

$$
\begin{aligned}
& W_{0}=a \times T_{4}+b \times T_{3}+c \times T_{2} \\
& W_{1}=a \times T_{5}+b \times T_{4}+c \times T_{3} \\
& W_{2}=a \times T_{6}+b \times T_{5}+c \times T_{4}
\end{aligned}
$$

we find that $a=10 W_{0}-15 W_{1}+6 W_{2}, b=37 W_{1}-24 W_{0}-15 W_{2}, c=15 W_{0}-$ $24 W_{1}+10 W_{2}$. The other equalities can be proved similarly.

Note that all the identities in the above Lemma can be proved by induction as well.

Next, we present a few basic relations between $\left\{W_{n}\right\}$ and $\left\{H_{n}\right\}$. 
Lemma 14. The following equalities are true:

(a) $\left(W_{0}-2 W_{1}+W_{2}\right) H_{n}=3 W_{n+4}-6 W_{n+3}+3 W_{n+2}$.

(b) $\left(W_{0}-2 W_{1}+W_{2}\right) H_{n}=3 W_{n+3}-6 W_{n+2}+3 W_{n+1}$.

(c) $\left(W_{0}-2 W_{1}+W_{2}\right) H_{n}=3 W_{n+2}-6 W_{n+1}+3 W_{n}$.

(d) $\left(W_{0}-2 W_{1}+W_{2}\right) H_{n}=3 W_{n+1}-6 W_{n}+3 W_{n-1}$.

(e) $\left(W_{0}-2 W_{1}+W_{2}\right) H_{n}=3 W_{n}-6 W_{n-1}+3 W_{n-2}$.

Now, we give a few basic relations between $\left\{W_{n}\right\}$ and $\left\{O_{n}\right\}$.

Lemma 15. The following equalities are true:

(a) $2 W_{n}=\left(10 W_{0}-15 W_{1}+6 W_{2}\right) O_{n+4}+\left(37 W_{1}-24 W_{0}-15 W_{2}\right) O_{n+3}+\left(15 W_{0}-\right.$ $\left.24 W_{1}+10 W_{2}\right) O_{n+2}$.

(b) $2 W_{n}=\left(6 W_{0}-8 W_{1}+3 W_{2}\right) O_{n+3}+\left(21 W_{1}-15 W_{0}-8 W_{2}\right) O_{n+2}+\left(10 W_{0}-\right.$ $\left.15 W_{1}+6 W_{2}\right) O_{n+1}$.

(c) $2 W_{n}=\left(3 W_{0}-3 W_{1}+W_{2}\right) O_{n+2}+\left(9 W_{1}-8 W_{0}-3 W_{2}\right) O_{n+1}+\left(6 W_{0}-8 W_{1}+\right.$ $\left.3 W_{2}\right) O_{n}$.

(d) $2 W_{n}=W_{0} O_{n+1}+\left(W_{1}-3 W_{0}\right) O_{n}+\left(3 W_{0}-3 W_{1}+W_{2}\right) O_{n-1}$.

(e) $2 W_{n}=W_{1} O_{n}+\left(W_{2}-3 W_{1}\right) O_{n-1}+W_{0} O_{n-2}$.

(f) $\left(W_{0}-2 W_{1}+W_{2}\right)^{3} O_{n}=-2\left(-3 W_{1}^{2}-W_{2}^{2}+W_{0} W_{1}+3 W_{1} W_{2}\right) W_{n+4}+2\left(-8 W_{1}^{2}-\right.$ $\left.3 W_{2}^{2}+3 W_{0} W_{1}-W_{0} W_{2}+9 W_{1} W_{2}\right) W_{n+3}+2\left(W_{0}^{2}+9 W_{1}^{2}+3 W_{2}^{2}-6 W_{0} W_{1}+\right.$ $\left.3 W_{0} W_{2}-10 W_{1} W_{2}\right) W_{n+2}$.

(g) $\left(W_{0}-2 W_{1}+W_{2}\right)^{3} O_{n}=-2\left(-W_{1}^{2}+W_{0} W_{2}\right) W_{n+3}+2\left(W_{0}^{2}-3 W_{0} W_{1}+\right.$ $\left.3 W_{0} W_{2}-W_{1} W_{2}\right) W_{n+2}-2\left(-3 W_{1}^{2}-W_{2}^{2}+W_{0} W_{1}+3 W_{1} W_{2}\right) W_{n+1}$.

(h) $\left(W_{0}-2 W_{1}+W_{2}\right)^{3} O_{n}=2\left(W_{0}^{2}+3 W_{1}^{2}-3 W_{0} W_{1}-W_{1} W_{2}\right) W_{n+2}-2\left(-W_{2}^{2}+\right.$ $\left.W_{0} W_{1}-3 W_{0} W_{2}+3 W_{1} W_{2}\right) W_{n+1}-2\left(-W_{1}^{2}+W_{0} W_{2}\right) W_{n}$. 
(i) $\left(W_{0}-2 W_{1}+W_{2}\right)^{3} O_{n}=2\left(3 W_{0}^{2}+9 W_{1}^{2}+W_{2}^{2}-10 W_{0} W_{1}+3 W_{0} W_{2}-\right.$ $\left.6 W_{1} W_{2}\right) W_{n+1}-2\left(3 W_{0}^{2}+8 W_{1}^{2}-9 W_{0} W_{1}+W_{0} W_{2}-3 W_{1} W_{2}\right) W_{n}+2\left(W_{0}^{2}+\right.$ $\left.3 W_{1}^{2}-3 W_{0} W_{1}-W_{1} W_{2}\right) W_{n-1}$.

(j) $\left(W_{0}-2 W_{1}+W_{2}\right)^{3} O_{n}=2\left(6 W_{0}^{2}+19 W_{1}^{2}+3 W_{2}^{2}-21 W_{0} W_{1}+8 W_{0} W_{2}-\right.$ $\left.15 W_{1} W_{2}\right) W_{n}-2\left(8 W_{0}^{2}+24 W_{1}^{2}+3 W_{2}^{2}-27 W_{0} W_{1}+9 W_{0} W_{2}-17 W_{1} W_{2}\right) W_{n-1}+$ $2\left(3 W_{0}^{2}+9 W_{1}^{2}+W_{2}^{2}-10 W_{0} W_{1}+3 W_{0} W_{2}-6 W_{1} W_{2}\right) W_{n-2}$.

Next, we present a few basic relations between $\left\{W_{n}\right\}$ and $\left\{p_{n}\right\}$.

Lemma 16. The following equalities are true:

(a) $27 W_{n}=\left(64 W_{0}-89 W_{1}+34 W_{2}\right) p_{n+4}+\left(229 W_{1}-158 W_{0}-89 W_{2}\right) p_{n+3}+$ $\left(103 W_{0}-158 W_{1}+64 W_{2}\right) p_{n+2}$.

(b) $27 W_{n}=\left(34 W_{0}-38 W_{1}+13 W_{2}\right) p_{n+3}+\left(109 W_{1}-89 W_{0}-38 W_{2}\right) p_{n+2}+\left(64 W_{0}-\right.$ $\left.89 W_{1}+34 W_{2}\right) p_{n+1}$.

(c) $27 W_{n}=\left(13 W_{0}-5 W_{1}+W_{2}\right) p_{n+2}+\left(25 W_{1}-38 W_{0}-5 W_{2}\right) p_{n+1}+\left(34 W_{0}-\right.$ $\left.38 W_{1}+13 W_{2}\right) p_{n}$.

(d) $27 W_{n}=\left(W_{0}+10 W_{1}-2 W_{2}\right) p_{n+1}+\left(10 W_{2}-23 W_{1}-5 W_{0}\right) p_{n}+\left(13 W_{0}-5 W_{1}+\right.$ $\left.W_{2}\right) p_{n-1}$.

(e) $27 W_{n}=\left(7 W_{1}-2 W_{0}+4 W_{2}\right) p_{n}+\left(10 W_{0}-35 W_{1}+7 W_{2}\right) p_{n-1}+\left(W_{0}+10 W_{1}-\right.$ $\left.2 W_{2}\right) p_{n-2}$.

(f) $\left(W_{0}-2 W_{1}+W_{2}\right)^{3} p_{n}=\left(2 W_{0}^{2}+21 W_{1}^{2}+7 W_{2}^{2}-13 W_{0} W_{1}+6 W_{0} W_{2}-\right.$ $\left.23 W_{1} W_{2}\right) W_{n+4}+\left(-6 W_{0}^{2}+37 W_{0} W_{1}-19 W_{0} W_{2}-56 W_{1}^{2}+63 W_{1} W_{2}-\right.$ $\left.19 W_{2}^{2}\right) W_{n+3}+\left(7 W_{0}^{2}+47 W_{1}^{2}+15 W_{2}^{2}-36 W_{0} W_{1}+19 W_{0} W_{2}-52 W_{1} W_{2}\right) W_{n+2}$.

(g) $\left(W_{0}-2 W_{1}+W_{2}\right)^{3} p_{n}=\left(7 W_{1}^{2}+2 W_{2}^{2}-2 W_{0} W_{1}-W_{0} W_{2}-6 W_{1} W_{2}\right) W_{n+3}+$ $\left(W_{0}^{2}-16 W_{1}^{2}-6 W_{2}^{2}+3 W_{0} W_{1}+W_{0} W_{2}+17 W_{1} W_{2}\right) W_{n+2}+\left(2 W_{0}^{2}+21 W_{1}^{2}+\right.$ $\left.7 W_{2}^{2}-13 W_{0} W_{1}+6 W_{0} W_{2}-23 W_{1} W_{2}\right) W_{n+1}$.

(h) $\left(W_{0}-2 W_{1}+W_{2}\right)^{3} p_{n}=\left(W_{0}^{2}+5 W_{1}^{2}-3 W_{0} W_{1}-2 W_{0} W_{2}-W_{1} W_{2}\right) W_{n+2}+$ $\left(2 W_{0}^{2}+W_{2}^{2}-7 W_{0} W_{1}+9 W_{0} W_{2}-5 W_{1} W_{2}\right) W_{n+1}+\left(7 W_{1}^{2}+2 W_{2}^{2}-2 W_{0} W_{1}-\right.$ $\left.W_{0} W_{2}-6 W_{1} W_{2}\right) W_{n}$. 
(i) $\left(W_{0}-2 W_{1}+W_{2}\right)^{3} p_{n}=\left(5 W_{0}^{2}+15 W_{1}^{2}+W_{2}^{2}-16 W_{0} W_{1}+3 W_{0} W_{2}-\right.$ $\left.8 W_{1} W_{2}\right) W_{n+1}+\left(-3 W_{0}^{2}+7 W_{0} W_{1}+5 W_{0} W_{2}-8 W_{1}^{2}-3 W_{1} W_{2}+2 W_{2}^{2}\right) W_{n}+$ $\left(W_{0}^{2}+5 W_{1}^{2}-3 W_{0} W_{1}-2 W_{0} W_{2}-W_{1} W_{2}\right) W_{n-1}$.

(j) $\left(W_{0}-2 W_{1}+W_{2}\right)^{3} p_{n}=\left(12 W_{0}^{2}+37 W_{1}^{2}+5 W_{2}^{2}-41 W_{0} W_{1}+14 W_{0} W_{2}-\right.$ $\left.27 W_{1} W_{2}\right) W_{n}+\left(-14 W_{0}^{2}+45 W_{0} W_{1}-11 W_{0} W_{2}-40 W_{1}^{2}+23 W_{1} W_{2}-\right.$ $\left.3 W_{2}^{2}\right) W_{n-1}+\left(5 W_{0}^{2}+15 W_{1}^{2}+W_{2}^{2}-16 W_{0} W_{1}+3 W_{0} W_{2}-8 W_{1} W_{2}\right) W_{n-2}$.

Now, we give a few basic relations between $\left\{T_{n}\right\}$ and $\left\{H_{n}\right\}$.

Lemma 17. The following equalities are true:

$$
\begin{aligned}
& H_{n}=3 T_{n+4}-6 T_{n+3}+3 T_{n+2}, \\
& H_{n}=3 T_{n+3}-6 T_{n+2}+3 T_{n+1}, \\
& H_{n}=3 T_{n+2}-6 T_{n+1}+3 T_{n}, \\
& H_{n}=3 T_{n+1}-6 T_{n}+3 T_{n-1}, \\
& H_{n}=3 T_{n}-6 T_{n-1}+3 T_{n-2},
\end{aligned}
$$

and so (since $H_{n}=3$ )

$$
\begin{aligned}
1 & =T_{n+4}-2 T_{n+3}+T_{n+2}, \\
1 & =T_{n+3}-2 T_{n+2}+T_{n+1}, \\
1 & =T_{n+2}-2 T_{n+1}+T_{n}, \\
1 & =T_{n+1}-2 T_{n}+T_{n-1}, \\
1 & =T_{n}-2 T_{n-1}+T_{n-2} .
\end{aligned}
$$

The following Remark gives a result on triangular numbers.

Remark 18. Note that for all integers $n$ and $m$, we have

$$
T_{n+m}-2 T_{n+m-1}+T_{n+m-2}=1 .
$$

Next, we present a few basic relations between $\left\{T_{n}\right\}$ and $\left\{O_{n}\right\}$. 
Lemma 19. The following equalities are true:

$$
\begin{aligned}
& 2 T_{n}=3 O_{n+4}-8 O_{n+3}+6 O_{n+2}, \\
& 2 T_{n}=O_{n+3}-3 O_{n+2}+3 O_{n+1}, \\
& 2 T_{n}=O_{n},
\end{aligned}
$$

and

$$
\begin{aligned}
O_{n} & =6 T_{n+4}-16 T_{n+3}+12 T_{n+2}, \\
O_{n} & =2 T_{n+3}-6 T_{n+2}+6 T_{n+1}, \\
O_{n} & =2 T_{n} .
\end{aligned}
$$

Now, we give a few basic relations between $\left\{T_{n}\right\}$ and $\left\{p_{n}\right\}$.

Lemma 20. The following equalities are true:

$$
\begin{aligned}
& 27 T_{n}=13 p_{n+4}-38 p_{n+3}+34 p_{n+2} \\
& 27 T_{n}=p_{n+3}-5 p_{n+2}+13 p_{n+1} \\
& 27 T_{n}=-2 p_{n+2}+10 p_{n+1}+p_{n} \\
& 27 T_{n}=4 p_{n+1}+7 p_{n}-2 p_{n-1} \\
& 27 T_{n}=19 p_{n}-14 p_{n-1}+4 p_{n-2}
\end{aligned}
$$

and

$$
\begin{aligned}
& p_{n}=15 T_{n+4}-38 T_{n+3}+26 T_{n+2}, \\
& p_{n}=7 T_{n+3}-19 T_{n+2}+15 T_{n+1}, \\
& p_{n}=2 T_{n+2}-6 T_{n+1}+7 T_{n}, \\
& p_{n}=T_{n}+2 T_{n-1} .
\end{aligned}
$$

Next, we present a few basic relations between $\left\{H_{n}\right\}$ and $\left\{O_{n}\right\}$. 
Lemma 21. The following equalities are true:

$$
\begin{aligned}
& 2 H_{n}=3 O_{n+4}-6 O_{n+3}+3 O_{n+2}, \\
& 2 H_{n}=3 O_{n+3}-6 O_{n+2}+3 O_{n+1}, \\
& 2 H_{n}=3 O_{n+2}-6 O_{n+1}+3 O_{n} \\
& 2 H_{n}=3 O_{n+1}-6 O_{n}+3 O_{n-1}, \\
& 2 H_{n}=3 O_{n}-6 O_{n-1}+3 O_{n-2},
\end{aligned}
$$

and so (since $H_{n}=3$ )

$$
\begin{aligned}
& 2=O_{n+4}-2 O_{n+3}+O_{n+2}, \\
& 2=O_{n+3}-2 O_{n+2}+O_{n+1}, \\
& 2=O_{n+2}-2 O_{n+1}+O_{n}, \\
& 2=O_{n+1}-2 O_{n}+O_{n-1}, \\
& 2=O_{n}-2 O_{n-1}+O_{n-2} .
\end{aligned}
$$

The following Remark gives a result on oblong numbers.

Remark 22. Note that for all integers $n$ and $m$, we have

$$
O_{n+m}-2 O_{n+m-1}+O_{n+m-2}=2 .
$$

Now, we give a few basic relations between $\left\{H_{n}\right\}$ and $\left\{p_{n}\right\}$.

Lemma 23. The following equalities are true

$$
\begin{aligned}
& H_{n}=p_{n+4}-2 p_{n+3}+p_{n+2}, \\
& H_{n}=p_{n+3}-2 p_{n+2}+p_{n+1}, \\
& H_{n}=p_{n+2}-2 p_{n+1}+p_{n}, \\
& H_{n}=p_{n+1}-2 p_{n}+p_{n-1}, \\
& H_{n}=p_{n}-2 p_{n-1}+p_{n-2},
\end{aligned}
$$


and so (since $H_{n}=3$ )

$$
\begin{aligned}
& 3=p_{n+4}-2 p_{n+3}+p_{n+2}, \\
& 3=p_{n+3}-2 p_{n+2}+p_{n+1} \\
& 3=p_{n+2}-2 p_{n+1}+p_{n}, \\
& 3=p_{n+1}-2 p_{n}+p_{n-1}, \\
& 3=p_{n}-2 p_{n-1}+p_{n-2} .
\end{aligned}
$$

The following Remark gives a result on pentagonal numbers.

Remark 24. Note that for all integers $n$ and $m$, we have

$$
p_{n+m}-2 p_{n+m-1}+p_{n+m-2}=3 .
$$

Next, we present a few basic relations between $\left\{O_{n}\right\}$ and $\left\{p_{n}\right\}$.

Lemma 25. The following equalities are true:

$$
\begin{aligned}
& 27 O_{n}=26 p_{n+4}-76 p_{n+3}+68 p_{n+2} \\
& 27 O_{n}=2 p_{n+3}-10 p_{n+2}+26 p_{n+1} \\
& 27 O_{n}=-4 p_{n+2}+20 p_{n+1}+2 p_{n} \\
& 27 O_{n}=8 p_{n+1}+14 p_{n}-4 p_{n-1} \\
& 27 O_{n}=38 p_{n}-28 p_{n-1}+8 p_{n-2},
\end{aligned}
$$

and

$$
\begin{aligned}
& 2 p_{n}=15 O_{n+4}-38 O_{n+3}+26 O_{n+2} \\
& 2 p_{n}=7 O_{n+3}-19 O_{n+2}+15 O_{n+1} \\
& 2 p_{n}=2 O_{n+2}-6 O_{n+1}+7 O_{n} \\
& 2 p_{n}=O_{n}+2 O_{n-1} .
\end{aligned}
$$

\section{$5 \quad$ Special Identities}

We now present a few special identities for the generalized Guglielmo sequence $\left\{W_{n}\right\}$. 
Theorem 26. (Catalan's identity of the generalized Guglielmo sequence) For all integers $n$ and $m$, the following identity holds:

$$
W_{n+m} W_{n-m}-W_{n}^{2}=\frac{m^{2}}{4} \Psi_{1}
$$

where

$\Psi_{1}=\left(m^{2}-2 n^{2}+2 n-1\right) W_{2}^{2}+4\left(m^{2}-2 n^{2}+4 n-4\right) W_{1}^{2}+\left(m^{2}-2 n^{2}+6 n-5\right) W_{0}^{2}-$ $4\left(m^{2}-2 n^{2}+3 n-2\right) W_{1} W_{2}+2\left(m^{2}-2 n^{2}+4 n-1\right) W_{0} W_{2}-4\left(m^{2}-2 n^{2}+5 n-4\right) W_{0} W_{1}$.

Proof. We use the identity $(2.2)$, (Binet's formula of $\left.W_{n}\right)$, i.e.,

$$
W_{n}=W_{0}+\frac{1}{2}\left(-W_{2}+4 W_{1}-3 W_{0}\right) n+\frac{1}{2}\left(W_{2}-2 W_{1}+W_{0}\right) n^{2} .
$$

As special cases of the above theorem, we have the following corollary.

Corollary 27. For all integers $n$ and $m$, the following identities hold:

(a) $T_{n+m} T_{n-m}-T_{n}^{2}=\frac{1}{4} m^{2}\left(m^{2}-2 n^{2}-2 n-1\right)$.

(b) $H_{n+m} H_{n-m}-H_{n}^{2}=0$.

(c) $O_{n+m} O_{n-m}-O_{n}^{2}=m^{2}\left(m^{2}-2 n^{2}-2 n-1\right)$.

(d) $p_{n+m} p_{n-m}-p_{n}^{2}=\frac{1}{4} m^{2}\left(9 m^{2}-18 n^{2}+6 n-1\right)$.

Note that for $m=1$ in Catalan's identity of the generalized Guglielmo sequence, we get the Cassini's identity for the generalized Guglielmo sequnce.

Theorem 28. (Cassini's identity of the generalized Guglielmo sequence) For all integers $n$, the following identity holds:

$$
W_{n+1} W_{n-1}-W_{n}^{2}=\frac{1}{2}\left(-n(n-1) W_{2}^{2}+2\left(-2 n^{2}+4 n-3\right) W_{1}^{2}-(n-1)(n-\right.
$$
2) $\left.W_{0}^{2}+2(2 n-1)(n-1) W_{1} W_{2}-2 n(n-2) W_{0} W_{2}+2(n-1)(2 n-3) W_{0} W_{1}\right)$.

As special cases of the above theorem, we have the following corollary.

Corollary 29. For all integers $n$, the following identities hold: 
(a) $T_{n+1} T_{n-1}-T_{n}^{2}=-\frac{1}{2} n(n+1)$.

(b) $H_{n+1} H_{n-1}-H_{n}^{2}=0$.

(c) $O_{n+1} O_{n-1}-O_{n}^{2}=-2 n(n+1)$.

(d) $p_{n+1} p_{n-1}-p_{n}^{2}=-\frac{1}{2}\left(9 n^{2}-3 n-4\right)$.

The d'Ocagne's identities can also be obtained by using 2.2. The next theorem presents d'Ocagne's identities of generalized Guglielmo sequence $\left\{W_{n}\right\}$.

Theorem 30. (d'Ocagne's identity) Let $n$ and $m$ be any integers. Then the following identities are true:

$W_{m+1} W_{n}-W_{m} W_{n+1}=-\frac{1}{2}(m-n)\left(m n W_{2}^{2}+2(2 m n-m-n+2) W_{1}^{2}+\right.$ $(n-1)(m-1) W_{0}^{2}+(-4 m n+m+n-1) W_{1} W_{2}+(2 m n-m-n-1) W_{0} W_{2}+$ $\left.(3 m+3 n-4 m n-3) W_{0} W_{1}\right)$.

Proof. Use the identity (2.2).

As special cases of the above theorem, we have the following corollary.

Corollary 31. For all integers n, the following identities hold:

(a) $T_{m+1} T_{n}-T_{m} T_{n+1}=-\frac{1}{2}(m-n)(n+1)(m+1)$.

(b) $H_{m+1} H_{n}-H_{m} H_{n+1}=0$.

(c) $O_{m+1} O_{n}-O_{m} O_{n+1}=-2(m-n)(n+1)(m+1)$.

(d) $p_{m+1} p_{n}-p_{m} p_{n+1}=-\frac{1}{2}(m-n)(9 m n+3 m+3 n-1)$.

\section{On the Recurrence Properties of Generalized Guglielmo Sequence}

Taking $r=3, s=-3, t=1$ in Theorem 6, we obtain the following Proposition. 
Proposition 32. For $n \in \mathbb{Z}$, generalized Guglielmo numbers (the case $r=3, s=$ $-3, t=1)$ have the following identity:

$$
\begin{aligned}
W_{-n} & =\left(W_{2 n}-H_{n} W_{n}+\frac{1}{2}\left(H_{n}^{2}-H_{2 n}\right) W_{0}\right) \\
& =W_{2 n}-3 W_{n}+3 W_{0} .
\end{aligned}
$$

Here $H_{n}=3$ and $H_{2 n}=3$ for all integers $n$. From the above Proposition and Corollary 8 , we have the following corollary which gives the connection between the special cases of generalized Guglielmo sequence at the positive index and the negative index: for triangular, triangular-Lucas, oblong and pentagonal numbers: take $W_{n}=T_{n}$ with $T_{0}=0, T_{1}=1, T_{2}=3$, take $W_{n}=H_{n}$ with $H_{0}=3, H_{1}=$ $3, H_{2}=3, W_{n}=O_{n}$ with $O_{0}=0, O_{1}=2, O_{2}=6$, and $W_{n}=p_{n}$ with $p_{0}=0, p_{1}=$ $1, p_{2}=5$, respectively. Note that in this case $H_{n}:=H_{n}$.

Corollary 33. For $n \in \mathbb{Z}$, we have the following recurrence relations:

(a) triangular sequence:

$$
T_{-n}=T_{2 n}-3 T_{n}
$$

(b) triangular-Lucas sequence:

$$
H_{-n}=3
$$

(c) oblong sequence:

$$
O_{-n}=O_{2 n}-3 O_{n}
$$

(d) pentagonal sequence:

$$
p_{-n}=p_{2 n}-3 p_{n}
$$

\section{Sum Formulas}

\subsection{Sums of Terms with Positive Subscripts:}

The following Theorem presents some formulas of of generalized Guglielmo numbers with indices in arithmetic progression. 
Theorem 34. For all integers $m$ and $j$, we have the following sum formulas:

$\sum_{k=0}^{n} W_{m k+j}=\frac{1}{12}(n+1)\left(\left(2 m^{2} n^{2}+m^{2} n+6 j m n-3 m n+6 j^{2}-6 j\right) W_{2}-2\left(2 m^{2} n^{2}+\right.\right.$ $\left.m^{2} n+6 j m n-6 m n+6 j^{2}-12 j\right) W_{1}+\left(2 m^{2} n^{2}+m^{2} n+6 j m n-9 m n+6 j^{2}-18 j+\right.$ 12) $\left.W_{0}\right)$.

Proof. Use the Binet's formula of generalized Guglielmo numbers, i.e.,

$$
W_{n}=W_{0}+\frac{1}{2}\left(-W_{2}+4 W_{1}-3 W_{0}\right) n+\frac{1}{2}\left(W_{2}-2 W_{1}+W_{0}\right) n^{2} .
$$

The following proposition presents some formulas of generalized Guglielmo numbers with positive subscripts.

Proposition 35. For $n \geq 0$, we have the following formulas:

(a) $\sum_{k=0}^{n} W_{k}=\frac{1}{6}(n+1)\left(n(n-1) W_{2}-n(2 n-5) W_{1}+\left(n^{2}-4 n+6\right) W_{0}\right)$.

(b) $\sum_{k=0}^{n} W_{2 k}=\frac{1}{6}(n+1)\left(n(4 n-1) W_{2}-8 n(n-1) W_{1}+\left(4 n^{2}-7 n+6\right) W_{0}\right)$.

(c) $\sum_{k=0}^{n} W_{2 k+1}=\frac{1}{6}(n+1)\left(n(4 n+5) W_{2}-2\left(4 n^{2}+2 n-3\right) W_{1}+n(4 n-1) W_{0}\right)$.

Proof. Take $m=1, j=0 ; m=2, j=0$ and $m=2, j=1$, respectively, in Theorem 34 .

From Theorem 34, we have the following corollary.

Corollary 36. For all integers $m$ and $j$, we have the following sum formulas:
(a) $\sum_{k=0}^{n} T_{m k+j}=\frac{1}{12}(n+1)\left(m^{2} n+2 m^{2} n^{2}+6 j m n+3 m n+6 j^{2}+6 j\right)$.
(b) $\sum_{k=0}^{n} H_{m k+j}=3(n+1)$.
(c) $\sum_{k=0}^{n} O_{m k+j}=\frac{1}{6}(n+1)\left(2 m^{2} n^{2}+m^{2} n+6 j m n+3 m n+6 j^{2}+6 j\right)$.
(d) $\sum_{k=0}^{n} p_{m k+j}=\frac{1}{4}(n+1)\left(2 m^{2} n^{2}+m^{2} n+6 j m n-m n+6 j^{2}-2 j\right)$. 
From the last Proposition 35 (or using Corollary 36), we have the following corollary which gives sum formulas of triangular numbers (take $W_{n}=T_{n}$ with $\left.T_{0}=0, T_{1}=1, T_{2}=3\right)$.

Corollary 37. For $n \geq 0$ we have the following formulas:

(a) $\sum_{k=0}^{n} T_{k}=\frac{1}{6} n(n+2)(n+1)$.

(b) $\sum_{k=0}^{n} T_{2 k}=\frac{1}{6} n(4 n+5)(n+1)$.

(c) $\sum_{k=0}^{n} T_{2 k+1}=\frac{1}{6}(n+1)(n+2)(4 n+3)$.

Taking $W_{n}=H_{n}$ with $H_{0}=3, H_{1}=3, H_{2}=3$ in the last Proposition 35 (or using Corollary 36), we have the following corollary which presents sum formulas of triangular-Lucas numbers.

Corollary 38. For $n \geq 0$ we have the following formulas:

(a) $\sum_{k=0}^{n} H_{k}=3(n+1)$.

(b) $\sum_{k=0}^{n} H_{2 k}=3(n+1)$.

(c) $\sum_{k=0}^{n} H_{2 k+1}=3(n+1)$.

From the last Proposition 35 (or using Corollary 36), we have the following corollary which gives sum formulas of oblong numbers (take $W_{n}=O_{n}$ with $O_{0}=$ $\left.0, O_{1}=2, O_{2}=6\right)$.

Corollary 39. For $n \geq 0$ we have the following formulas:

(a) $\sum_{k=0}^{n} O_{k}=\frac{1}{3} n(n+2)(n+1)$.

(b) $\sum_{k=0}^{n} O_{2 k}=\frac{1}{3} n(4 n+5)(n+1)$.

(c) $\sum_{k=0}^{n} O_{2 k+1}=\frac{1}{3}(n+1)(n+2)(4 n+3)$.

Taking $W_{n}=p_{n}$ with $p_{0}=0, p_{1}=1, p_{2}=5$ in the last Proposition 35 (or using Corollary 36 ), we have the following corollary which presents sum formulas of pentagonal numbers. 
Corollary 40. For $n \geq 0$ we have the following formulas:

(a) $\sum_{k=0}^{n} p_{k}=\frac{1}{2} n^{2}(n+1)$.

(b) $\sum_{k=0}^{n} p_{2 k}=\frac{1}{2} n(4 n+1)(n+1)$.

(c) $\sum_{k=0}^{n} p_{2 k+1}=\frac{1}{2}(n+1)\left(7 n+4 n^{2}+2\right)$.

\subsection{Sums of Squares of Terms with Positive Subscripts}

The following Theorem presents some formulas of of generalized Guglielmo numbers with indices in arithmetic progression.

Theorem 41. For all integers $m, j, p$ and $q$, we have the following sum formulas:

(a)

$$
\sum_{k=0}^{n} W_{m k+j}^{2}=\frac{n+1}{120} \Delta
$$

where

$\Delta=\left(5 m^{2} n-m^{4} n+10 m^{2} n^{2}-15 m^{3} n^{2}-15 m^{3} n^{3}+m^{4} n^{2}+9 m^{4} n^{3}+6 m^{4} n^{4}+\right.$ $30 j^{2}-60 j^{3}+30 j^{4}+60 j^{2} m^{2} n^{2}-30 j m^{2} n-90 j^{2} m n+60 j^{3} m n-60 j m^{2} n^{2}+$ $\left.30 j^{2} m^{2} n+30 j m^{3} n^{2}+30 j m^{3} n^{3}+30 j m n\right) W_{2}^{2}+4\left(20 m^{2} n-m^{4} n+40 m^{2} n^{2}-\right.$ $30 m^{3} n^{2}-30 m^{3} n^{3}+m^{4} n^{2}+9 m^{4} n^{3}+6 m^{4} n^{4}+120 j^{2}-120 j^{3}+30 j^{4}+60 j^{2} m^{2} n^{2}-$ $60 j m^{2} n-180 j^{2} m n+60 j^{3} m n-120 j m^{2} n^{2}+30 j^{2} m^{2} n+30 j m^{3} n^{2}+30 j m^{3} n^{3}+$ $120 j m n) W_{1}^{2}+\left(-360 j+65 m^{2} n-m^{4} n+130 m^{2} n^{2}-45 m^{3} n^{2}-45 m^{3} n^{3}+\right.$ $m^{4} n^{2}+9 m^{4} n^{3}+6 m^{4} n^{4}-180 m n+390 j^{2}-180 j^{3}+30 j^{4}+60 j^{2} m^{2} n^{2}-$ $90 j m^{2} n-270 j^{2} m n+60 j^{3} m n-180 j m^{2} n^{2}+30 j^{2} m^{2} n+30 j m^{3} n^{2}+30 j m^{3} n^{3}+$ $390 j m n+120) W_{0}^{2}-2\left(20 m^{2} n-2 m^{4} n+40 m^{2} n^{2}-45 m^{3} n^{2}-45 m^{3} n^{3}+\right.$ $2 m^{4} n^{2}+18 m^{4} n^{3}+12 m^{4} n^{4}+120 j^{2}-180 j^{3}+60 j^{4}+120 j^{2} m^{2} n^{2}-90 j m^{2} n-$ $\left.270 j^{2} m n+120 j^{3} m n-180 j m^{2} n^{2}+60 j^{2} m^{2} n+60 j m^{3} n^{2}+60 j m^{3} n^{3}+120 j m n\right)$ $W_{1} W_{2}+2\left(-60 j+25 m^{2} n-m^{4} n+50 m^{2} n^{2}-30 m^{3} n^{2}-30 m^{3} n^{3}+m^{4} n^{2}+\right.$ $9 m^{4} n^{3}+6 m^{4} n^{4}-30 m n+150 j^{2}-120 j^{3}+30 j^{4}+60 j^{2} m^{2} n^{2}-60 j m^{2} n-$ $\left.180 j^{2} m n+60 j^{3} m n-120 j m^{2} n^{2}+30 j^{2} m^{2} n+30 j m^{3} n^{2}+30 j m^{3} n^{3}+150 j m n\right)$ $W_{0} W_{2}-2\left(-240 j+80 m^{2} n-2 m^{4} n+160 m^{2} n^{2}-75 m^{3} n^{2}-75 m^{3} n^{3}+2 m^{4} n^{2}+\right.$ 
$18 m^{4} n^{3}+12 m^{4} n^{4}-120 m n+480 j^{2}-300 j^{3}+60 j^{4}+120 j^{2} m^{2} n^{2}-150 j m^{2} n-$ $\left.450 j^{2} m n+120 j^{3} m n-300 j m^{2} n^{2}+60 j^{2} m^{2} n+60 j m^{3} n^{2}+60 j m^{3} n^{3}+480 j m n\right)$ $W_{0} W_{1}$.

(b)

$$
\sum_{k=0}^{n} W_{m k+j} W_{p k+q}=\frac{n+1}{240} \Omega
$$

where

$\Omega=\left(20 j^{2} n^{2} p^{2}+10 j^{2} n p^{2}+60 j^{2} n p q-30 j^{2} n p+60 j^{2} q^{2}-60 j^{2} q+30 j m n^{3} p^{2}+\right.$ $30 j m n^{2} p^{2}+80 j m n^{2} p q-40 j m n^{2} p+40 j m n p q-20 j m n p+60 j m n q^{2}-60 j m n q-$ $20 j n^{2} p^{2}-10 j n p^{2}-60 j n p q+30 j n p-60 j q^{2}+60 j q+12 m^{2} n^{4} p^{2}+18 m^{2} n^{3} p^{2}+$ $30 m^{2} n^{3} p q-15 m^{2} n^{3} p+2 m^{2} n^{2} p^{2}+30 m^{2} n^{2} p q-15 m^{2} n^{2} p+20 m^{2} n^{2} q^{2}-20$ $m^{2} n^{2} q-2 m^{2} n p^{2}+10 m^{2} n q^{2}-10 m^{2} n q-15 m n^{3} p^{2}-15 m n^{2} p^{2}-40 m n^{2} p q+$ $\left.20 m n^{2} p-20 m n p q+10 m n p-30 m n q^{2}+30 m n q\right) W_{2}^{2}+8\left(10 j^{2} n^{2} p^{2}+5 j^{2} n p^{2}+\right.$ $30 j^{2} n p q-30 j^{2} n p+30 j^{2} q^{2}-60 j^{2} q+15 j m n^{3} p^{2}+15 j m n^{2} p^{2}+40 j m n^{2} p q-$ $40 j m n^{2} p+20 j m n p q-20 j m n p+30 j m n q^{2}-60 j m n q-20 j n^{2} p^{2}-10 j n p^{2}-60$ $j n p q+60 j n p-60 j q^{2}+120 j q+6 m^{2} n^{4} p^{2}+9 m^{2} n^{3} p^{2}+15 m^{2} n^{3} p q-15 m^{2} n^{3} p+$ $m^{2} n^{2} p^{2}+15 m^{2} n^{2} p q-15 m^{2} n^{2} p+10 m^{2} n^{2} q^{2}-20 m^{2} n^{2} q-m^{2} n p^{2}+5 m^{2} n q^{2}-$ $10 m^{2} n q-15 m n^{3} p^{2}-15 m n^{2} p^{2}-40 m n^{2} p q+40 m n^{2} p-20 m n p q+20 m$ $\left.n p-30 m n q^{2}+60 m n q\right) W_{1}^{2}+\left(20 j^{2} n^{2} p^{2}+10 j^{2} n p^{2}+60 j^{2} n p q-90 j^{2} n p+60 j^{2} q^{2}-\right.$ $180 j^{2} q+120 j^{2}+30 j m n^{3} p^{2}+30 j m n^{2} p^{2}+80 j m n^{2} p q-120 j m n^{2} p+40 j m n p q-$ $60 j m n p+60 j m n q^{2}-180 j m n q+120 j m n-60 j n^{2} p^{2}-30 j n p^{2}-180 j n p q+270 j n$ $p-180 j q^{2}+540 j q-360 j+12 m^{2} n^{4} p^{2}+18 m^{2} n^{3} p^{2}+30 m^{2} n^{3} p q-45 m^{2} n^{3} p+$ $2 m^{2} n^{2} p^{2}+30 m^{2} n^{2} p q-45 m^{2} n^{2} p+20 m^{2} n^{2} q^{2}-60 m^{2} n^{2} q+40 m^{2} n^{2}-2 m^{2} n p^{2}+$ $10 m^{2} n q^{2}-30 m^{2} n q+20 m^{2} n-45 m n^{3} p^{2}-45 m n^{2} p^{2}-120 m n^{2} p q+180 m n^{2} p-$ $60 m n p q+90 m n p-90 m n q^{2}+270 m n q-180 m n+40 n^{2} p^{2}+20 n p^{2}+120 n p q-180$ $\left.n p+120 q^{2}-360 q+240\right) W_{0}^{2}+2\left(-40 j^{2} n^{2} p^{2}-20 j^{2} n p^{2}-120 j^{2} n p q+90 j^{2}\right.$ $n p-120 j^{2} q^{2}+180 j^{2} q-60 j m n^{3} p^{2}-60 j m n^{2} p^{2}-160 j m n^{2} p q+120 j m n^{2} p-$ $80 j m n p q+60 j m n p-120 j m n q^{2}+180 j m n q+60 j n^{2} p^{2}+30 j n p^{2}+180 j n p q-$ $120 j n p+180 j q^{2}-240 j q-24 m^{2} n^{4} p^{2}-36 m^{2} n^{3} p^{2}-60 m^{2} n^{3} p q+45 m^{2} n^{3} p-$ $4 m^{2} n^{2} p^{2}-60 m^{2} n^{2} p q+45 m^{2} n^{2} p-40 m^{2} n^{2} q^{2}+60 m^{2} n^{2} q+4 m^{2} n p^{2}-20 m^{2} n q^{2}+$ $30 m^{2} n q+45 m n^{3} p^{2}+45 m n^{2} p^{2}+120 m n^{2} p q-80 m n^{2} p+60 m n p q-40 m n p+$ 
$\left.90 m n q^{2}-120 m n q\right) W_{1} W_{2}+4\left(10 j^{2} n^{2} p^{2}+5 j^{2} n p^{2}+30 j^{2} n p q-30 j^{2} n p+\right.$ $30 j^{2} q^{2}-60 j^{2} q+30 j^{2}+15 j m n^{3} p^{2}+15 j m n^{2} p^{2}+40 j m n^{2} p q-40 j m n^{2} p+$ $20 j m n p q-20 j m n p+30 j m n q^{2}-60 j m n q+30 j m n-20 j n^{2} p^{2}-10 j n p^{2}-$ $60 j n p q+45 j n p-60 j q^{2}+90 j q-30 j+6 m^{2} n^{4} p^{2}+9 m^{2} n^{3} p^{2}+15 m^{2} n^{3} p q-$ $15 m^{2} n^{3} p+m^{2} n^{2} p^{2}+15 m^{2} n^{2} p q-15 m^{2} n^{2} p+10 m^{2} n^{2} q^{2}-20 m^{2} n^{2} q+10 m^{2} n^{2}-$ $m^{2} n p^{2}+5 m^{2} n q^{2}-10 m^{2} n q+5 m^{2} n-15 m n^{3} p^{2}-15 m n^{2} p^{2}-40 m n^{2} p q+$ $30 m n^{2} p-20 m n p q+15 m n p-30 m n q^{2}+45 m n q-15 m n+10 n^{2} p^{2}+5 n p^{2}+30$ $\left.n p q-15 n p+30 q^{2}-30 q\right) W_{0} W_{2}+2\left(-40 j^{2} n^{2} p^{2}-20 j^{2} n p^{2}-120 j^{2} n p q+150\right.$ $j^{2} n p-120 j^{2} q^{2}+300 j^{2} q-120 j^{2}-60 j m n^{3} p^{2}-60 j m n^{2} p^{2}-160 j m n^{2} p q+$ $200 j m n^{2} p-80 j m n p q+100 j m n p-120 j m n q^{2}+300 j m n q-120 j m n+$ $100 j n^{2} p^{2}+50 j n p^{2}+300 j n p q-360 j n p+300 j q^{2}-720 j q+240 j-24 m^{2} n^{4} p^{2}-$ $36 m^{2} n^{3} p^{2}-60 m^{2} n^{3} p q+75 m^{2} n^{3} p-4 m^{2} n^{2} p^{2}-60 m^{2} n^{2} p q+75 m^{2} n^{2} p-40 m^{2} n^{2}$ $q^{2}+100 m^{2} n^{2} q-40 m^{2} n^{2}+4 m^{2} n p^{2}-20 m^{2} n q^{2}+50 m^{2} n q-20 m^{2} n+75 m n^{3} p^{2}+$ $75 m n^{2} p^{2}+200 m n^{2} p q-240 m n^{2} p+100 m n p q-120 m n p+150 m n q^{2}-360 m n q+$ $\left.120 m n-40 n^{2} p^{2}-20 n p^{2}-120 n p q+120 n p-120 q^{2}+240 q\right) W_{0} W_{1}$.

Proof. Use the Binet's formula of generalized Guglielmo numbers, i.e.,

$$
W_{n}=W_{0}+\frac{1}{2}\left(-W_{2}+4 W_{1}-3 W_{0}\right) n+\frac{1}{2}\left(W_{2}-2 W_{1}+W_{0}\right) n^{2} .
$$

From Theorem 41, we have the following corollary.

Corollary 42. For all integers $m, j, p$ and $q$, we have the following sum formulas:

(a)

(i)

$$
\sum_{k=0}^{n} T_{m k+j}^{2}=\frac{n+1}{120} \Delta_{1}
$$

where

$$
\begin{aligned}
& \Delta_{1}=5 m^{2} n-m^{4} n+10 m^{2} n^{2}+15 m^{3} n^{2}+15 m^{3} n^{3}+m^{4} n^{2}+9 m^{4} n^{3}+ \\
& 6 m^{4} n^{4}+30 j^{2}+60 j^{3}+30 j^{4}+60 j^{2} m^{2} n^{2}+30 j m^{2} n+90 j^{2} m n+60 j^{3} m n+60 \\
& j m^{2} n^{2}+30 j^{2} m^{2} n+30 j m^{3} n^{2}+30 j m^{3} n^{3}+30 j m n .
\end{aligned}
$$


(ii)

$$
\sum_{k=0}^{n} T_{m k+j} T_{p k+q}=\frac{n+1}{240} \Omega_{1}
$$

where

$\Omega_{1}=20 j^{2} n^{2} p^{2}+10 j^{2} n p^{2}+60 j^{2} n p q+30 j^{2} n p+60 j^{2} q^{2}+60 j^{2} q+$ $30 j m n^{3} p^{2}+30 j m n^{2} p^{2}+80 j m n^{2} p q+40 j m n^{2} p+40 j m n p q+20 j m n p+$ $60 j m n q^{2}+60 j m n q+20 j n^{2} p^{2}+10 j n p^{2}+60 j n p q+30 j n p+60 j q^{2}+60 j q+$ $12 m^{2} n^{4} p^{2}+18 m^{2} n^{3} p^{2}+30 m^{2} n^{3} p q+15 m^{2} n^{3} p+2 m^{2} n^{2} p^{2}+30 m^{2} n^{2} p q+$ $15 m^{2} n^{2} p+20 m^{2} n^{2} q^{2}+20 m^{2} n^{2} q-2 m^{2} n p^{2}+10 m^{2} n q^{2}+10 m^{2} n q+15 m$ $n^{3} p^{2}+15 m n^{2} p^{2}+40 m n^{2} p q+20 m n^{2} p+20 m n p q+10 m n p+30 m n q^{2}+$ $30 m n q$.

(b)

(i)

$$
\sum_{k=0}^{n} H_{m k+j}^{2}=9(n+1) .
$$

(ii)

$$
\sum_{k=0}^{n} H_{m k+j} H_{p k+q}=9(n+1) .
$$

(c)

(i)

$$
\sum_{k=0}^{n} O_{m k+j}^{2}=\frac{n+1}{120} \Delta_{2}
$$

where

$$
\begin{aligned}
& \Delta_{2}=4\left(5 m^{2} n-m^{4} n+10 m^{2} n^{2}+15 m^{3} n^{2}+15 m^{3} n^{3}+m^{4} n^{2}+9 m^{4} n^{3}+\right. \\
& 6 m^{4} n^{4}+30 j^{2}+60 j^{3}+30 j^{4}+60 j^{2} m^{2} n^{2}+30 j m^{2} n+90 j^{2} m n+60 j^{3} m n+ \\
& \left.60 j m^{2} n^{2}+30 j^{2} m^{2} n+30 j m^{3} n^{2}+30 j m^{3} n^{3}+30 j m n\right) .
\end{aligned}
$$

(ii)

$$
\sum_{k=0}^{n} O_{m k+j} O_{p k+q}=\frac{n+1}{240} \Omega_{2}
$$


where

$\Omega_{2}=4\left(20 j^{2} n^{2} p^{2}+10 j^{2} n p^{2}+60 j^{2} n p q+30 j^{2} n p+60 j^{2} q^{2}+60 j^{2} q+\right.$ $30 j m n^{3} p^{2}+30 j m n^{2} p^{2}+80 j m n^{2} p q+40 j m n^{2} p+40 j m n p q+20 j m n p+$ $60 j m n q^{2}+60 j m n q+20 j n^{2} p^{2}+10 j n p^{2}+60 j n p q+30 j n p+60 j q^{2}+60 j q+$ $12 m^{2} n^{4} p^{2}+18 m^{2} n^{3} p^{2}+30 m^{2} n^{3} p q+15 m^{2} n^{3} p+2 m^{2} n^{2} p^{2}+30 m^{2} n^{2} p q+$ $15 m^{2} n^{2} p+20 m^{2} n^{2} q^{2}+20 m^{2} n^{2} q-2 m^{2} n p^{2}+10 m^{2} n q^{2}+10 m^{2} n q+15 m$ $n^{3} p^{2}+15 m n^{2} p^{2}+40 m n^{2} p q+20 m n^{2} p+20 m n p q+10 m n p+30 m n q^{2}+$ $30 m n q)$.

(d)

(i)

$$
\sum_{k=0}^{n} p_{m k+j}^{2}=\frac{n+1}{120} \Delta_{3}
$$

where

$\Delta_{3}=5 m^{2} n-9 m^{4} n+10 m^{2} n^{2}-45 m^{3} n^{2}-45 m^{3} n^{3}+9 m^{4} n^{2}+81 m^{4} n^{3}+$ $54 m^{4} n^{4}+30 j^{2}-180 j^{3}+270 j^{4}+540 j^{2} m^{2} n^{2}-90 j m^{2} n-270 j^{2} m n+$ $540 j^{3} m n-180 j m^{2} n^{2}+270 j^{2} m^{2} n+270 j m^{3} n^{2}+270 j m^{3} n^{3}+30 j m n$.

(ii)

$$
\sum_{k=0}^{n} p_{m k+j} p_{p k+q}=\frac{n+1}{240} \Omega_{3}
$$

where

$\Omega_{3}=-180 j q^{2}-180 j^{2} q+540 j^{2} q^{2}+60 j q+180 j^{2} n^{2} p^{2}+18 m^{2} n^{2} p^{2}+$ $162 m^{2} n^{3} p^{2}+180 m^{2} n^{2} q^{2}+108 m^{2} n^{4} p^{2}-30 j n p^{2}-90 j^{2} n p+20 m n^{2} p-$ $90 m n q^{2}-30 m^{2} n q-60 j n^{2} p^{2}+90 j^{2} n p^{2}-45 m n^{2} p^{2}-18 m^{2} n p^{2}-$ $45 m^{2} n^{2} p-45 m n^{3} p^{2}-45 m^{2} n^{3} p+90 m^{2} n q^{2}-60 m^{2} n^{2} q+30 j n p+10 m n p+$ $30 m n q-120 j m n^{2} p+540 j m n q^{2}+540 j^{2} n p q-120 m n^{2} p q+270 j m n^{2} p^{2}+$ $270 j m n^{3} p^{2}+270 m^{2} n^{2} p q+270 m^{2} n^{3} p q-60 j m n p-180 j m n q-180 j n p q-$ $60 m n p q+720 j m n^{2} p q+360 j m n p q$.

From the last proposition, we have the following Corollary which gives sum formulas of triangular numbers. 
Corollary 43. For $n \geq 0$, triangular numbers have the following properties:

(a) $\sum_{k=0}^{n} T_{k}^{2}=\frac{1}{60} n(n+1)(n+2)\left(3 n^{2}+6 n+1\right)$.

(b) $\sum_{k=0}^{n} T_{k+1} T_{k}=\frac{1}{40} n(n+1)(n+2)(n+3)(2 n+3)$.

(c) $\sum_{k=0}^{n} T_{k+2} T_{k}=\frac{1}{20} n(n+1)(n+2)(n+3)(n+4)$.

Proof. For (a), take $m=1, j=0$ in Corollary 42 (a) (i) and for (b) and (c), take $m=1, j=1, p=1, q=0$ and $m=1, j=2, p=1, q=0$, respectively, in Corollary 42 (a) (ii).

From the last proposition, we have the following Corollary which presents sum formulas of triangular-Lucas numbers.

Corollary 44. For $n \geq 0$, triangular-Lucas numbers have the following properties:

(a) $\sum_{k=0}^{n} H_{k}^{2}=9(n+1)$.

(b) $\sum_{k=0}^{n} H_{k+1} H_{k}=9(n+1)$.

(c) $\sum_{k=0}^{n} H_{k+2} H_{k}=9(n+1)$.

Proof. For (a), take $m=1, j=0$ in Corollary 42 (b) (i) and for (b) and (c), take $m=1, j=1, p=1, q=0$ and $m=1, j=2, p=1, q=0$, respectively, in Corollary 42 (b) (ii).

From the last proposition, we have the following corollary which gives sum formulas of oblong numbers.

Corollary 45. For $n \geq 0$, oblong numbers have the following properties:

(a) $\sum_{k=0}^{n} O_{k}^{2}=\frac{1}{15} n(n+1)(n+2)\left(3 n^{2}+6 n+1\right)$.

(b) $\sum_{k=0}^{n} O_{k+1} O_{k}=\frac{1}{10} n(n+1)(n+2)(n+3)(2 n+3)$.

(c) $\sum_{k=0}^{n} O_{k+2} O_{k}=\frac{1}{5} n(n+1)(n+2)(n+3)(n+4)$. 
Proof. For (a), take $m=1, j=0$ in Corollary 42 (c) (i) and for (b) and (c), take $m=1, j=1, p=1, q=0$ and $m=1, j=2, p=1, q=0$, respectively, in Corollary 42 (c) (ii).

From the last proposition, we have the following corollary which presents sum formulas of pentagonal numbers.

Corollary 46. For $n \geq 0$, pentagonal numbers have the following properties:

(a) $\sum_{k=0}^{n} p_{k}^{2}=\frac{1}{60} n(n+1)\left(27 n^{3}+18 n^{2}-13 n-2\right)$.

(b) $\sum_{k=0}^{n} p_{k+1} p_{k}=\frac{1}{120} n(n+1)(n+2)\left(54 n^{2}+63 n-17\right)$.

(c) $\sum_{k=0}^{n} p_{k+2} p_{k}=\frac{1}{60} n(n+1)\left(27 n^{3}+153 n^{2}+212 n-32\right)$.

Proof. For (a), take $m=1, j=0$ in Corollary 42 (d) (i) and for (b) and (c), take $m=1, j=1, p=1, q=0$ and $m=1, j=2, p=1, q=0$, respectively, in Corollary 42 (d) (ii).

\section{Matrices Related With Generalized Guglielmo numbers}

We define the square matrix $A$ of order 3 as:

$$
A=\left(\begin{array}{ccc}
3 & -3 & 1 \\
1 & 0 & 0 \\
0 & 1 & 0
\end{array}\right)
$$

such that $\operatorname{det} A=1$. From (2.1) we have

$$
\left(\begin{array}{c}
W_{n+2} \\
W_{n+1} \\
W_{n}
\end{array}\right)=\left(\begin{array}{ccc}
3 & -3 & 1 \\
1 & 0 & 0 \\
0 & 1 & 0
\end{array}\right)\left(\begin{array}{c}
W_{n+1} \\
W_{n} \\
W_{n-1}
\end{array}\right)
$$


and from (1.5) (or using (8.1) and induction) we have

$$
\left(\begin{array}{c}
W_{n+2} \\
W_{n+1} \\
W_{n}
\end{array}\right)=\left(\begin{array}{ccc}
3 & -3 & 1 \\
1 & 0 & 0 \\
0 & 1 & 0
\end{array}\right)^{n}\left(\begin{array}{l}
W_{2} \\
W_{1} \\
W_{0}
\end{array}\right) .
$$

If we take $W=T$ in (8.1) we have

$$
\left(\begin{array}{c}
T_{n+2} \\
T_{n+1} \\
T_{n}
\end{array}\right)=\left(\begin{array}{ccc}
3 & -3 & 1 \\
1 & 0 & 0 \\
0 & 1 & 0
\end{array}\right)\left(\begin{array}{c}
T_{n+1} \\
T_{n} \\
T_{n-1}
\end{array}\right) .
$$

We also define

$$
B_{n}=\left(\begin{array}{ccc}
T_{n+1} & -3 T_{n}+T_{n-1} & T_{n} \\
T_{n} & -3 T_{n-1}+T_{n-2} & T_{n-1} \\
T_{n-1} & -3 T_{n-2}+T_{n-3} & T_{n-2}
\end{array}\right)
$$

and

$$
C_{n}=\left(\begin{array}{ccc}
W_{n+1} & -3 W_{n}+W_{n-1} & W_{n} \\
W_{n} & -3 W_{n-1}+W_{n-2} & W_{n-1} \\
W_{n-1} & -3 W_{n-2}+W_{n-3} & W_{n-2}
\end{array}\right)
$$

Theorem 47. For all integers $m, n \geq 0$, we have

(a) $B_{n}=A^{n}$

(b) $C_{1} A^{n}=A^{n} C_{1}$

(c) $C_{n+m}=C_{n} B_{m}=B_{m} C_{n}$.

Proof. Take $r=3, s=-3, t=1$ in Soykan 21. Theorem 5.1].

Some properties of matrix $A^{n}$ can be given as

$$
A^{n}=3 A^{n-1}-3 A^{n-2}+A^{n-3}
$$

and

$$
A^{n+m}=A^{n} A^{m}=A^{m} A^{n}
$$


and

$$
\operatorname{det}\left(A^{n}\right)=1
$$

for all integers $m$ and $n$.

Corollary 48. For all integers $n$, we have the following formulas for the triangular, triangular-Lucas, oblong and pentagonal numbers.

(a) Triangular Numbers.

$$
\begin{aligned}
A^{n} & =\left(\begin{array}{ccc}
3 & -3 & 1 \\
1 & 0 & 0 \\
0 & 1 & 0
\end{array}\right)^{n}=\left(\begin{array}{ccc}
T_{n+1} & -3 T_{n}+T_{n-1} & T_{n} \\
T_{n} & -3 T_{n-1}+T_{n-2} & T_{n-1} \\
T_{n-1} & -3 T_{n-2}+T_{n-3} & T_{n-2}
\end{array}\right) \\
& =\left(\begin{array}{ccc}
\frac{1}{2}(n+1)(n+2) & -n(n+2) & \frac{1}{2} n(n+1) \\
\frac{1}{2} n(n+1) & -(n-1)(n+1) & \frac{1}{2} n(n-1) \\
\frac{1}{2} n(n-1) & -n(n-2) & \frac{1}{2}(n-1)(n-2)
\end{array}\right) .
\end{aligned}
$$

(b) Oblong Numbers.

$$
\begin{aligned}
A^{n} & =\frac{1}{2}\left(\begin{array}{ccc}
O_{n+1} & -3 O_{n}+O_{n-1} & O_{n} \\
O_{n} & -3 O_{n-1}+O_{n-2} & O_{n-1} \\
O_{n-1} & -3 O_{n-2}+O_{n-3} & O_{n-2}
\end{array}\right) \\
& =\left(\begin{array}{ccc}
\frac{1}{2}(n+1)(n+2) & -n(n+2) & \frac{1}{2} n(n+1) \\
\frac{1}{2} n(n+1) & -(n-1)(n+1) & \frac{1}{2} n(n-1) \\
\frac{1}{2} n(n-1) & -n(n-2) & \frac{1}{2}(n-1)(n-2)
\end{array}\right) .
\end{aligned}
$$

(c) Pentagonal Numbers. 


$$
\begin{gathered}
A^{n=} \frac{1}{27}\left(\begin{array}{ccc}
-2 p_{n+3}+10 p_{n+2}+p_{n+1} & 6 p_{n+2}-32 p_{n+1}+7 p_{n}+p_{n-1} \\
-2 p_{n+2}+10 p_{n+1}+p_{n} & 6 p_{n+1}-32 p_{n}+7 p_{n-1}+p_{n-2} \\
-2 p_{n+1}+10 p_{n}+p_{n-1} & 6 p_{n}-32 p_{n-1}+7 p_{n-2}+p_{n-3} \\
-2 p_{n+2}+10 p_{n+1}+p_{n} \\
-2 p_{n+1}+10 p_{n}+p_{n-1} \\
-2 p_{n}+10 p_{n-1}+p_{n-2}
\end{array}\right) \\
=\left(\begin{array}{ccc}
\frac{1}{2}(n+1)(n+2) & -n(n+2) & \frac{1}{2} n(n+1) \\
\frac{1}{2} n(n+1) & -(n-1)(n+1) & \frac{1}{2} n(n-1) \\
\frac{1}{2} n(n-1) & -n(n-2) & \frac{1}{2}(n-1)(n-2)
\end{array}\right) .
\end{gathered}
$$

Proof. (a) It is given in Theorem 47 (a).

(b) Note that

$$
2 T_{n}=O_{n} .
$$

Using the last equation and (a), we get required result.

(c) Note that, from Lemma 20, we know that

$$
27 T_{n}=-2 p_{n+2}+10 p_{n+1}+p_{n} .
$$

Using the last equation and (a), we get required result.

Theorem 49. For all integers $m, n$, we have

$$
\begin{aligned}
W_{n+m} & =W_{n} T_{m+1}+W_{n-1}\left(-3 T_{m}+T_{m-1}\right)+W_{n-2} T_{m} \\
& =W_{n} T_{m+1}+\left(-3 W_{n-1}+W_{n-2}\right) T_{m}+W_{n-1} T_{m-1}
\end{aligned}
$$

Proof. Take $r=3, s=-3, t=1$ in Soykan 21, Theorem 5.2.].

By Lemma 13, we know that

$$
\begin{aligned}
\left(W_{0}-2 W_{1}+W_{2}\right)^{3} T_{m}= & \left(W_{0}^{2}+3 W_{1}^{2}-3 W_{0} W_{1}-W_{1} W_{2}\right) W_{m+2} \\
& +\left(W_{2}^{2}-W_{0} W_{1}+3 W_{0} W_{2}-3 W_{1} W_{2}\right) W_{m+1} \\
& +\left(W_{1}^{2}-W_{0} W_{2}\right) W_{m}
\end{aligned}
$$


so 8.3 can be written in the following form

$$
\begin{aligned}
& \left(W_{0}-2 W_{1}+W_{2}\right)^{3} W_{n+m} \\
= & W_{n}\left(\left(W_{0}^{2}+3 W_{1}^{2}-3 W_{0} W_{1}-W_{1} W_{2}\right) W_{m+3}\right. \\
& \left.+\left(W_{2}^{2}-W_{0} W_{1}+3 W_{0} W_{2}-3 W_{1} W_{2}\right) W_{m+2}+\left(W_{1}^{2}-W_{0} W_{2}\right) W_{m+1}\right) \\
& +\left(-3 W_{n-1}+W_{n-2}\right)\left(\left(W_{0}^{2}+3 W_{1}^{2}-3 W_{0} W_{1}-W_{1} W_{2}\right) W_{m+2}\right. \\
& \left.+\left(W_{2}^{2}-W_{0} W_{1}+3 W_{0} W_{2}-3 W_{1} W_{2}\right) W_{m+1}+\left(W_{1}^{2}-W_{0} W_{2}\right) W_{m}\right) \\
& +W_{n-1}\left(\left(W_{0}^{2}+3 W_{1}^{2}-3 W_{0} W_{1}-W_{1} W_{2}\right) W_{m+1}\right. \\
& \left.+\left(W_{2}^{2}-W_{0} W_{1}+3 W_{0} W_{2}-3 W_{1} W_{2}\right) W_{m}+\left(W_{1}^{2}-W_{0} W_{2}\right) W_{m-1}\right) .
\end{aligned}
$$

Corollary 50. For all integers $m, n$, we have

$$
\begin{aligned}
T_{n+m} & =T_{n} T_{m+1}+T_{n-1}\left(-3 T_{m}+T_{m-1}\right)+T_{n-2} T_{m}, \\
H_{n+m} & =H_{n} T_{m+1}+H_{n-1}\left(-3 T_{m}+T_{m-1}\right)+H_{n-2} T_{m}, \\
O_{n+m} & =O_{n} T_{m+1}+O_{n-1}\left(-3 T_{m}+T_{m-1}\right)+O_{n-2} T_{m}, \\
p_{n+m} & =p_{n} T_{m+1}+p_{n-1}\left(-3 T_{m}+T_{m-1}\right)+p_{n-2} T_{m},
\end{aligned}
$$

and

$$
\begin{aligned}
2 O_{m+n}= & O_{n} O_{m+1}+O_{m}\left(O_{n-2}-3 O_{n-1}\right)+O_{m-1} O_{n-1} \\
27 p_{m+n}= & p_{m+2}\left(4 p_{n}+7 p_{n-1}-2 p_{n-2}\right)+p_{m+1}\left(7 p_{n}-35 p_{n-1}+10 p_{n-2}\right) \\
& -p_{m}\left(2 p_{n}-10 p_{n-1}-p_{n-2}\right) .
\end{aligned}
$$

Note that since $H_{n+m}=H_{n}=H_{n-1}=H_{n-2}=3$, we write $H_{n+m}=$ $H_{n} T_{m+1}+H_{n-1}\left(-3 T_{m}+T_{m-1}\right)+H_{n-2} T_{m}$ as

$$
1=T_{m+1}+\left(-3 T_{m}+T_{m-1}\right)+T_{m}
$$

i.e.,

$$
1=T_{m+1}-2 T_{m}+T_{m-1} .
$$

Taking $m=n$ in the last corollary we obtain the following identities:

$$
\begin{aligned}
T_{2 n} & =T_{n-1}^{2}+\left(T_{n+1}-3 T_{n-1}+T_{n-2}\right) T_{n}, \\
O_{2 n} & =O_{n} T_{n+1}+O_{n-1}\left(-3 T_{n}+T_{n-1}\right)+O_{n-2} T_{n}, \\
p_{2 n} & =p_{n} T_{n+1}+p_{n-1}\left(-3 T_{n}+T_{n-1}\right)+p_{n-2} T_{n},
\end{aligned}
$$


and

$$
\begin{aligned}
2 O_{2 n}= & O_{n} O_{n+1}+O_{n}\left(O_{n-2}-3 O_{n-1}\right)+O_{n-1} O_{n-1}, \\
27 p_{2 n}= & p_{n+2}\left(4 p_{n}+7 p_{n-1}-2 p_{n-2}\right)+p_{n+1}\left(7 p_{n}-35 p_{n-1}+10 p_{n-2}\right) \\
& -p_{n}\left(2 p_{n}-10 p_{n-1}-p_{n-2}\right) .
\end{aligned}
$$

\section{References}

[1] I. Bruce, A modified Tribonacci sequence, Fibonacci Quarterly 22(3) (1984), 244-246.

[2] R. M. Castillo, A survey on triangular number, factorial and some associated numbers, Indian Journal of Science and Technology 9(41) (2016). https://doi.org/10.17485/ijst/2016/v9i41/85182

[3] M. Catalani, Identities for Tribonacci-related sequences, 2012. arXiv:math/0209179

[4] E. Choi, Modular Tribonacci numbers by matrix method, Journal of the Korean Society of Mathematical Education Series B: Pure and Applied Mathematics 20(3) (2013), 207-221. https://doi.org/10.7468/jksmeb.2013.20.3.207

[5] J. H. Conway and R. K. Guy, The Book of Numbers, New York: Copernicus, 1996.

[6] M. Elia, Derived sequences, the Tribonacci recurrence and cubic forms, Fibonacci Quarterly 39(2) (2001), 107-115.

[7] M. C. Er, Sums of Fibonacci numbers by matrix methods, Fibonacci Quarterly 22(3) (1984), 204-207.

[8] A. S. Garge and A. A. Shirali, Triangular numbers, Resonance 17 (2012), 672-681. https://doi.org/10.1007/s12045-012-0074-z

[9] D. Kalman, Generalized Fibonacci numbers By matrix methods, Fibonacci Quarterly 20(1) (1982), 73-76.

[10] W. R. Knorr, The Evolution of the Euclidean Elements, Dordrecht-Boston, Mass.: D. Reidel Publishing Co., 1975.

[11] K. Kuhapatanakul and A. G. Shannon, Binomial coefficients and triangular numbers, European Journal of Mathematics and Statistics 2(3) (2021). http://dx.doi.org/10.24018/ejmath.2021.2.3.31 
[12] M. Lemma, D. Lemaitre and R. Edwards, The triangular numbers in actions, International Journal of Mathematics 4(11) (2021), 1-11.

[13] P. Y. Lin, De Moivre-Type identities for the Tribonacci numbers, Fibonacci Quarterly 26 (1988), 131-134.

[14] T. E. Moore, Some observations on Oblong numbers, Mathematical Medley 39(1) (2013), 29-32.

[15] S. Pethe, Some identities for Tribonacci sequences, Fibonacci Quarterly 26(2) (1988), 144-151.

[16] A. Scott, T. Delaney and V. Hoggatt, Jr., The Tribonacci sequence, Fibonacci Quarterly 15(3) (1977), 193-200.

[17] A. Shannon, Tribonacci numbers and Pascal's pyramid, Fibonacci Quarterly 15(3) (1977), 268 and 275.

[18] N. J. A. Sloane, editor, The On-Line Encyclopedia of Integer Sequences, published electronically at https://oeis.org

[19] Y. Soykan, Simson identity of generalized $m$-step Fibonacci numbers, Int. J. Adv. Appl. Math. and Mech. 7(2) (2019), 45-56.

[20] Y. Soykan, Tribonacci and Tribonacci-Lucas Sedenions, Mathematics 7(1) (2019), 74. https://doi.org/10.3390/math7010074

[21] Y. Soykan, A study on generalized $(r, s, t)$-numbers, MathLAB Journal 7 (2020), 101-129.

[22] Y. Soykan, On the recurrence properties of generalized Tribonacci sequence, Earthline Journal of Mathematical Sciences 6(2) (2021), 253-269. https://doi.org/10.34198/ejms.6221.253269

[23] W. Spickerman, Binet's formula for the Tribonacci sequence, Fibonacci Quarterly 20 (1982), 118-120.

[24] C. C. Yalavigi, Properties of Tribonacci numbers, Fibonacci Quarterly 10(3) (1972), 231-246. 
[25] N. Yilmaz and N. Taskara, Tribonacci and Tribonacci-Lucas numbers via the determinants of special matrices, Applied Mathematical Sciences 8(39) (2014), 1947-1955. http://dx.doi.org/10.12988/ams.2014.4270

[26] E.W. Weisstein, Polygonal Number, in: From MathWorld-A Wolfram Web Resource, https://mathworld.wolfram.com/PolygonalNumber.html

[27] E. W. Weisstein, Pentagonal Number, in: From MathWorld-A Wolfram Web Resource, https://mathworld.wolfram.com/PentagonalNumber.html

[28] Wikipedia, Pentagonal Number, in: From Wikipedia-A Web Resource, https://en.wikipedia.org/wiki/Pentagonal_number

[29] Wikipedia, Polygonal Number, in: From Wikipedia-A Web Resource, https://en.wikipedia.org/wiki/Polygonal_number

This is an open access article distributed under the terms of the Creative Commons Attribution License (http://creativecommons.org/licenses/by/4.0/), which permits unrestricted, use, distribution and reproduction in any medium, or format for any purpose, even commercially provided the work is properly cited. 